\title{
Odor Encoding as an Active, Dynamical Process: Experiments, Computation, and Theory
}

\author{
Gilles Laurent, ${ }^{1}$ Mark Stopfer, ${ }^{1}$ Rainer W Friedrich, ${ }^{1}$ \\ Misha I Rabinovich, ${ }^{2}$ Alexander Volkovskii, ${ }^{2}$ \\ and Henry DI Abarbanel, ${ }^{2,3}$ \\ ${ }^{1}$ California Institute of Technology, Division of Biology 139-74, Pasadena, California \\ 91125; e-mail: laurentg@caltech.edu,stopfer@cns.caltech.edu,friedric@cns.caltech.edu \\ ${ }^{2}$ University of California San Diego, Institute for Nonlinear Science, La Jolla, California \\ 92093-0402; e-mail: mrabinovich@ucsd.edu,avolkovskii@ucsd.edu, \\ hdia@hamilton.ucsd.edu \\ ${ }^{3}$ Department of Physics and Marine Physical Laboratory, Scripps Institution of \\ Oceanography, University of California San Diego, La Jolla, California 92093
}

\begin{abstract}
Key Words olfaction, olfactory bulb, antennal lobe, learning, dynamical systems, oscillations

- Abstract We examine early olfactory processing in the vertebrate and insect olfactory systems, using a computational perspective. What transformations occur between the first and second olfactory processing stages? What are the causes and consequences of these transformations? To answer these questions, we focus on the functions of olfactory circuit structure and on the role of time in odor-evoked integrative processes. We argue that early olfactory relays are active and dynamical networks, whose actions change the format of odor-related information in very specific ways, so as to refine stimulus identification. Finally, we introduce a new theoretical framework ("winnerless competition") for the interpretation of these data.
\end{abstract}

\section{INTRODUCTION}

The olfactory brain converts generally complex air- or water-borne chemical mixtures into singular signatures, experienced as vivid percepts. Such transformations are achieved by way of only a few brain stations-olfactory circuits are shallower than their visual and auditory counterparts - and are strongly tied to emotions and to memories acquired through other modalities. Understanding olfactory coding is thus an ambitious enterprise whose scope goes much beyond that of this review. We focus here on the sensory transformations accomplished 
by the first two processing stages, that is, olfactory receptors and postsynaptic structures.

As with any sense, understanding olfaction first requires defining the problems it has evolved to solve (Attneave 1954, Barlow 1969): segmenting an odor into its various constituents, as a chemist might do, does not appear to be one of these functions (Lawless 1997, Cain \& Potts 1996). Rather, olfaction is a synthetic sense par excellence. Olfaction enables pattern learning, storage, recognition, tracking, or localization and attaches "meaning" to these patterns. By meaning we imply the richer set of associations acquired through other senses as well as hedonic (pleasant/unpleasant) and emotional valence-both of which have no physical reality outside the brain. Each one of these tasks needs to be better defined; recognition, for example, encompasses at least categorization, identification, and separation. The abilities to categorize and to identify a priori each imply very different kinds of processing; for example, categorization disregards small differences, whereas identification emphasizes them. We show how a single circuit can in fact accomplish both, through the use of dynamics.

We should also exploit our understanding of the physics of odors. In vision, much attention has been given to the statistics of natural images (Field 1987, 1994; Olshausen \& Field 1996; Rudderman 1994). Correlations across space and time make natural images highly nonstochastic. Also, the spatial-frequency $(f)$ content of a natural image, be it a face or a landscape, obeys a $1 / f^{\alpha}$ distribution (at any scale), implying large areas of low contrast cut by edges whose frequency of occurrence decreases as sharpness increases. Knowing these rules helps us understand or predict the existence of specific spatial filters in early vision. Have equivalent rules been extracted from our knowledge of natural odors? It seems not. Many natural odors (such as families of flower fragrances) consist of similar combinations of compounds, implying the existence of natural correlations across volatile molecules; however, it seems as well that many unnatural combinations concocted in the laboratory evoke odors that are just as real or learnable. This impression remains anecdotal; we do not know of systematic investigations of odor statistics or of an animal's ability to learn and recognize composite odors. Help may eventually come from studies of olfactory receptor functional "specificity" (Buck 1995), although the complexity of chemical space may preclude such simple analysis. Some physical features of odors, however, are well known. Odor plumes, for instance, discretize stimulus delivery (Murlis et al 1992). This temporal structure is reinforced by odor-sampling behavior, which is usually repetitive (e.g. sniffing, appendage flicking, and casting behavior [Mellon 1997, Murlis et al 1992]). We examine the consequences of such intermittency on odor representations and their implications for olfactory codes. Finally, whereas sounds and images are typically updated rapidly, thus conveying information at potentially high rates that are exploitable for high-throughput communication, odors change more slowly. Olfaction is generally a low-temporal (and often spatial)-bandwidth sense. This means that time can be used as a coding dimension for the representation of nontemporal features of odors (Laurent 1999). 


\section{GOALS AND CONCEPTUAL FRAMEWORK}

The cloning of odorant receptors and the structure and mapping of early olfactory circuits have been comprehensively reviewed over the last decade (Axel 1995; Boeckh \& Tolbert 1993; Buck 1996; Hildebrand \& Shepherd 1997; Mombaerts et al 1996b; Satou 1990; Scott et al 1993; Shepherd 1993, 1994; Shipley \& Ennis 1996). We here focus on a computational view of odor encoding within the vertebrate main olfactory bulb $(\mathrm{OB})$ and the analogous circuit in insects, the main antennal lobe (AL), excluding macroglomerular or specialized pheromonal centers (Dulac 1997, Keverne 1999). The OB and AL are organized according to similar anatomical principles (Hildebrand \& Shepherd 1997); these similarities are only reinforced by physiological observations. Our approach thus tends to emphasize potential principles rather than peculiarities.

The foundation of this review is that early olfactory circuits should be studied as a system. The $\mathrm{OB}$ and $\mathrm{AL}$ form richly interconnected circuits whose global mode of action is captured accurately by neither static nor isolated samples (e.g. neither anatomical studies nor single-cell recordings); these circuits cannot be viewed as passive relays of odor-related information. Because olfactory-neuron responses are complex and correlated across space, a study of odor encoding must take into account these interactions and their distributed consequences. These consequences unfold over time; the early olfactory system behaves as an active nonstationary system; its dynamics occur on multiple time scales. Hence, the transfer to olfaction of functional models developed with other sensory systems is often inappropriate.

For example, analyses of visual, auditory, or somatosensory physiology rely heavily on the concept of the tuning curve, applied to single-neuron studies (e.g. Hubel \& Wiesel 1968). This approach implies a scalar description of the stimulus (e.g. position or frequency; i.e. in a space with one or a few independent dimensions) and a one-dimensional scoring of a neuron's response (usually mean firing rate). While empirically powerful, both descriptions are ill adapted to much of olfactory physiology. One reason is that the relevant olfactory stimulus dimensions are likely to be numerous and difficult to order in relation to one another; a second reason is that neuronal response profiles are usually temporally complex, and the information they carry is not conveyed fully by firing rates. Moreover, because most "classical" studies focused, for technical reasons, on unitary recordings, their significance is always examined in a framework in which the single neuron is the relevant informational entity: the unspoken implication is that "whoever" reads activity within the brain does it either one neuron at a time or by simple spatial or temporal averaging of a population's activity (Shadlen \& Newsome 1995), disregarding interneuronal correlations. Our results indicate that this is not appropriate for olfactory codes (Laurent 1996, Laurent et al 1996, MacLeod et al 1998, Stopfer et al 1997, Wehr \& Laurent 1996). We argue that understanding olfactory coding requires a shift to a different framework, in which relevance is measured globally and over time. 
Walter Freeman's many seminal contributions to the development of a dynamical perspective on olfaction (Freeman 1978, 1992, 2000; Freeman \& Skarda 1985) must be recognized here. Our approach and interpretations, however, differ from Freeman's in at least three important ways. The first lies in the nature of the data. While we recognize the importance of macro- or mesoscopic signals (e.g. EEGs and field potentials) as experimental tools, we believe that they are not of the appropriate scale for analysis. The spatiotemporal phenomena that cause recognizable features in field potentials (e.g. local synchronization and nonstationary behavior) are indeed functionally relevant; but field potentials are only "shadows" of underlying distributed but precise neural-activity patterns, which need to be deciphered. The second difference lies in our theoretical model of population behavior. "Winnerless competition," introduced later, depends to a significant extent on a neuron-resolution-mechanistic understanding of odor signal processing. The third difference is that our experimental approach, using small olfactory systems (insects and fish), tries to separate stimulus-evoked activity from centrifugal "higher" influences providing contextual information. Our goal, illustrated here, is to understand the "unsupervised" sensory formatting of odor representations by early olfactory circuits first, although we agree that expectation influences odor-evoked neural activity (Pager 1983, Kay \& Freeman 1998, Kay \& Laurent 1999).

\section{DISTRIBUTED, CLUSTERED REPRESENTATIONS BY AFFERENT ARRAYS}

\section{Olfactory-Receptor-Neuron Tuning and Combinatorial Codes}

Odor encoding is a spatially distributed process. Since the classical work of Adrian (1942, 1950, 1953), it has been shown repeatedly and with increasing clarity that single odorants activate neural activity over a wide area of the nasal epithelium and its postsynaptic target neuropils (Cinelli \& Kauer 1992, Duchamp 1982, Duchamp-Viret \& Duchamp 1997, Friedrich \& Korsching 1997, Joerges et al 1997, Kauer 1987, Leveteau \& MacLeod 1966, Moulton 1967, Rubin \& Katz 1999, Stewart et al 1979). Such broad activation is now, through remarkable advances in the molecular biology of odorant receptors (Buck \& Axel 1991, Buck 1996), understandable in terms of olfactory receptor neuron (ORN) tuning and axon projections; single ORNs express a limited number of odorant receptor types (probably a single one in mammals), and all ORNs expressing the same receptor type converge to one or a few glomeruli in their target areas (Bozza \& Kauer 1997, Malnic et al 1999, Mombaerts et al 1996a, Ressler et al 1993, Vassar et al 1994, Wang et al 1998). Because single odors can activate broad overlapping regions, it is clear that individual neurons - and thus possibly the receptor proteins themselves - can be activated by many odorants, including ones that belong to different chemical families, underlying different odor qualities. Calcium imaging on dissociated ORNs (Bozza \& Kauer 1997, Malnic et al 1999) and older 
studies in situ (Duchamp et al 1974, Getchell 1986, Kang \& Caprio 1995) are consistent with such results. The molecular/structural mechanisms underlying receptor-ligand recognition remain poorly understood. In conclusion, odors, be they mono- or multimolecular, generally activate an array of receptor types and, hence, a distributed population of glomeruli. Different odors activate different combinations of glomeruli; the odor identity code across ORNs thus has a critical combinatorial component.

\section{Clustering Within Odor Representations by Olfactory Receptor Neurons}

Many studies have illustrated the distributed nature of odor-evoked activity (Buck 1996); the clearest demonstration of distributed representations across ORNs, however, comes from recent calcium- and voltage-sensitive imaging experiments in zebrafish (Friedrich \& Korsching 1997, 1998). Indeed, while glomeruli are generally described as the functional units of the early olfactory system, often overlooked is that they contain projections from afferent fibers (ORNs), intrinsic neurons (periglomerular cells), and output neurons (mitral and tufted cells [M/TCs]). Describing glomerular activity without assessing the respective contributions of pre- and postsynaptic elements precludes any understanding of the computations carried out there. Friedrich \& Korsching's (1997) studies are significant in that only ORNs were labeled, ensuring that patterns of glomerular activation reported only ORN activity (although the possible contribution of presynaptic inhibition and thus of intrinsic neurons cannot be excluded). They showed that natural amino acid odorants activate overlapping combinations of glomeruli (i.e. ORN populations) and that odors represented by similar combinations share chemical features (e.g. acidic, basic, or long-chain neutral). Hence, the molecular underpinnings of olfactory transduction result in redundant (overlapping) odor representations (representation clusters) by afferent arrays (see also Duchamp-Viret \& Duchamp 1997). Less direct evidence suggests that this is true also for other species (Bozza \& Kauer 1997, Imamura et al 1992, Joerges et al 1997, Katoh et al 1993). Note that such clustering might provide a physical substrate for perceptual odor categorization. We see later that dynamical processing within the OB alters this initial clustered format.

\section{OLFACTORY-RECEPTOR-NEURON SCATTERING, AXONAL CONVERGENCE, AND NOISE REDUCTION}

ORNs that express the same receptor are, at least in fish and rodents (Ngai et al 1993, Ressler et al 1993, Weth et al 1996), distributed randomly within wide and overlapping zones across the nasal epithelium. Much interest is now focused on how the axons of these ORNs converge on the same glomeruli in the OB (or AL) during development (Lin et al 2000, Zheng et al 2000). This distributed structure 
itself, however, raises an important question: Are there computational gains in scattering rather than grouping idiotypic ORNs? One advantage might be noise reduction. The reasoning is twofold. First, scattering ORNs across the receptive sheet ensures that global activity across the ORN array is minimally affected by local fluctuations; because air (or water) flow along turbinates is turbulent, noisy fluctuations in odor concentration affecting some receptors are unlikely to be correlated across the entire ORN array. Second, ORN-to-OB/AL output neuron convergence ratios are generally very large $(\sim 1000: 1$ in mammals and 100:1 in many insects). A mitral cell or its insect analog (projection neuron [PN]) thus forms its responses to odors from very large numbers of converging inputs. These two conditions (uncorrelated noise and convergence) should ensure that postsynaptic averaging increases signal-to-noise ratios. Amphibian ORNs can display periodic synchronization, independent of downstream activity (Dorries \& Kauer 2000, Ottoson 1959). Although indicating correlated modulation of ORN input, this does not contradict the above hypothesis, provided that noise from external sources remains uncorrelated across the ORN population. We shall see below that stimulus-noise reduction is highly desirable if further processing seeks to amplify the differences between overlapping representations (within-cluster patterns) for fine odor discrimination.

\section{LATERAL CONNECTIONS WITHIN THE OLFACTORY BULB/ANTENNAL LOBE}

\section{Connections}

Olfactory-Receptor-Neuron Output Once odors activate groups of ORNs and their corresponding glomerular targets, information does not simply flow through the OB/AL, channeled directly by projection neurons to downstream areas. Rather, glomerular target sites and projection neurons form widespread lateral connections within the $\mathrm{OB} / \mathrm{AL}$. In the vertebrate $\mathrm{OB}$, for example, M/TCs sometimes possess dendrites in several glomeruli (e.g. rabbit and turtle [Mori et al 1981, 1983]) and thus combine inputs with different chemical sources. Such patterns are common in lower vertebrates (Dryer \& Graziadei 1994, Nieuwenhuys 1967) and in the AL of many insects (e.g. locusts and wasps) (Laurent 1996, Masson \& Mustaparta 1990).

Local Inhibition Local inhibitory neurons (granule cells [GCs] and periglomerular cells in vertebrates and local neurons [LNs] in insects) interconnect excitatory projection cells via excitatory inhibitory pathways. Resulting interactions can be very local, such as those mediated by periglomerular cells between neighboring glomeruli in the mammalian $\mathrm{OB}$, or widespread, such as those mediated by GCs between M/TCs with deep secondary dendrites in the external plexiform layer (Dryer \& Graziadei 1994). GC-mediated pathways may indeed link M/TCs whose somata and input glomeruli lie millimeters apart (Shipley \& Ennis 1996). 
The strength of this functional linkage, however, may vary with distance, owing to biophysical properties of M/TC secondary dendrites (Chen et al 1997). Note also that, in rodents at least, ORN projections bifurcate to two hemi-OBs; it is not clear yet whether the glomerular topology is identical in both. If local glomerular neighborhoods differ between the two halves, yet more combinations of lateral interactions between different input types can be created. If hemi-OBs are identical, GC-mediated interactions across the border between the hemi-OBs should allow interglomerular interactions that are different from those between the glomeruli within each hemi-OB. Insect inhibitory LNs often (and in certain species, always) have widespread arborizations, giving them access, in principle, to almost any neuron in the system, e.g. in Drosophila melanogaster (Heisenberg et al 1985) and in locusts (Laurent 1996).

Other Pathways Interactions between projection cells or between LNs allow for more complex polysynaptic pathways. In the turtle and mammalian OB for example, evidence exists for lateral long-lasting excitatory interactions between M/TCs (Aroniadou-Anderjaska et al 1999, Isaacson 1999, Nicoll 1971a, Nicoll \& Jahr 1982). In insects, PN-PN synaptic connections have been directly observed (Leitch \& Laurent 1996; Malun 1991a, 1991b). Similarly, GCs or LNs can inhibit each other (Leitch \& Laurent 1996, Shipley \& Ennis 1996), enabling disinhibition, that is, context-dependent excitation. Finally, output neurons may send axonal collaterals within the OB/AL, thus influencing other neurons directly or polysynaptically (Nicoll 1971b, Nicoll \& Jahr 1982, Gray \& Skinner 1988). In short, ORNs affect neurons other than their immediate targets in glomeruli, even if the underlying lateral circuits vary across species (Dryer \& Graziadei 1994). The OB (like the AL) is not a simple point-to-point relay (Kauer 1991). This observation underlies the dynamical framework introduced below.

\section{Functions}

The existence of GC-mediated lateral inhibition has been known for many decades; inhibitory neurons unquestionably make lateral contacts. We question, however, the common functional interpretation that these contacts serve to sharpen singleM/TC tuning through a process akin to retinal "lateral inhibition" (DeVries \& Baylor 1993, Mori \& Shepherd 1994). We find unconvincing the physiological evidence for such single-cell "sharpening" and the rationale for these connections' role (Imamura et al 1992, Katoh et al 1993, Mori et al 1992, Yokoi et al 1995) (see Laurent 1999). A "retinal" understanding of lateral inhibition, for example, predicts that cells "best tuned" to a given odor should be selected at the expense of suboptimally activated neurons (winner take all) over the duration of a stimulus. We found no evidence for such trends (RW Friedrich \& G Laurent, submitted manuscript). We also observe that an individual neuron often responds to many odors (hindering the fusion of anatomical and functional lateral inhibitions) and that responses are not stationary (see below). We thus propose a different interpretation-that contacts 
mediated by GCs and other neurons contribute together to a global reformatting of odor representations, in the form of a stimulus-dependent, temporal redistribution of activity across the OB/AL. One can thus think of lateral interactions as a means to sharpen odor representations by the population in a manner often not assessable from single-neuron data. We now review the supporting evidence.

\section{TEMPORAL FEATURES OF OLFACTORY RESPONSES}

\section{Historical Background}

Oscillations Adrian $(1942,1950)$ first reported the existence of oscillatory activity in the olfactory systems of anaesthetized mammals. Later studies (Freeman 2000, Freeman \& Skarda 1985, Gray \& Skinner 1988) showed that spontaneous or odor-driven oscillatory activity could contain complex frequency spectra. Recent physiological and imaging studies also revealed that the spatial coherence of oscillatory activity across or between olfactory areas can be complex (Lam et al 1999) and, in behaving animals, context dependent (Kay \& Freeman 1998). Odor-evoked oscillatory activity has since been observed in most animal classes, including mollusks (Gelperin \& Tank 1990), insects (Laurent \& Naraghi 1994), fish (Satou 1990), amphibians (Ottoson 1959), reptiles (Beuerman 1975), and mammals, including primates (Hughes \& Mazurowski 1962). Early computational studies indicated that lateral and reciprocal synapses between mitral cells (MCs) and GCs could generate the oscillatory patterning observed experimentally (Rall \& Shepherd 1968). Other studies predicted also that such connections should impose a $90^{\circ}$ average phase shift between MC and GC activity (Freeman 1975). The precise synaptic and biophysical mechanisms underlying or participating in oscillatory synchronization vary, and yet, odor-driven oscillations are ubiquitous in olfactory systems, including downstream areas, such as piriform and entorhinal cortices in mammals (Haberly 1990). As with other brain areas where stimulusevoked oscillations occur (Gray 1994, Singer \& Gray 1995), a major question emerges: Is oscillatory synchrony functionally relevant?

Slow Single-Unit Patterning Following Adrian's pioneering observations, many single-unit studies on mammals, fish, and amphibians revealed that MC responses to odors are not fully described by firing rates (Hamilton \& Kauer 1989, Kauer \& Moulton 1974, Macrides \& Chorover 1972, Meredith 1986, Wellis et al 1989). Extracellular recordings of OB units in anaesthetized rodents, for example, showed that neurons exhibit odor-dependent temporal discharge patterns relative to an imposed inhalation rhythm (Macrides \& Chorover 1972). The structure of the patterns evoked by one odor appeared to be more stable over the duration of an experiment than firing rates and was largely independent of the imposed inhalation schedule or the concentration. Intracellular recordings from salamander MCs 
documented the deep sculpting of their responses by phasic inhibition (Kauer \& Moulton 1974). Later intracellular studies on the insect pheromonal system (Burrows et al 1982) described similar features. Finally, voltage-sensitive dye imaging in salamanders provided macroscopic evidence for spatiotemporal patterns evoked by odors over large areas of the OB (Cinelli \& Kauer 1992, Cinelli et al 1995). As anticipated (Kauer 1991), "presumably, ... this spatio-temporal array [of MCs] now carries re-encoded information about the stimulus to the next level of integration, the olfactory cortices"... Our recent work in insects and fish builds on these seminal studies. It links together many past and some new observations, attempts to provide a functional understanding of this re-encoding, and tests its relevance.

\section{The Locust Model System}

Insects provide an accessible model for forms of odor processing observed throughout a broad range of animals (Hildebrand \& Shepherd 1997).

Circuits The insect main AL contains the processes of three main neuron populations: ORN axon terminals, LNs, and PNs. LNs are inhibitory, although their population is, in some species, heterogeneous. PNs are excitatory and project to two areas: the mushroom body, an area involved in multimodal processing (Heisenberg 1998, Strausfeld et al 1998) and associative memory (including olfactory; Heisenberg et al 1985, Menzel 1987), and the lateral protocerebral lobe. Details about these pathways vary somewhat with species (Masson \& Mustaparta 1990). In addition, the AL contains the terminals of aminergic or peptidergic neuromodulatory neurons, whose projections are generally widespread within the brain (Hammer 1993, Sun et al 1993) and whose effects on associative learning can be critical (Hammer \& Menzel 1995). The AL has a glomerular architecture, but the number of glomeruli varies (e.g. $\sim 50$ in D. melanogaster and $\sim 1000$ in locusts or wasps). Species with few large glomeruli are described as macroglomerular; the others are microglomerular. Accordingly, PN projections can be uniglomerular (as in macroglomerular species, e.g. D. melanogaster, bees, and cockroaches) or multiglomerular (e.g. wasps and locusts). These morphological subtypes are not correlated with categories of physiological output: PN oscillatory synchronization and slow response patterning (see below) are observed equally in micro- and macroglomerular species (Heinbockel et al 1998, Laurent \& Naraghi 1994, Stopfer et al 1997). In locusts, the AL contains the terminals of $\sim 90,000$ ORNs, $\sim 300$ LNs, and 830 PNs. LN projections are axonless and extend over the entire AL. Each PN has a planar dendritic tree with 10-20 radial dendrites each ending in one or two glomeruli (Laurent et al 1996). Neither LNs nor PNs appear to express intrinsic oscillatory properties (Laurent \& Davidowitz 1994). Upon natural stimulation, odor-specific subgroups of LNs and PNs become activated. Their responses contain several interlocked features, reviewed below. 

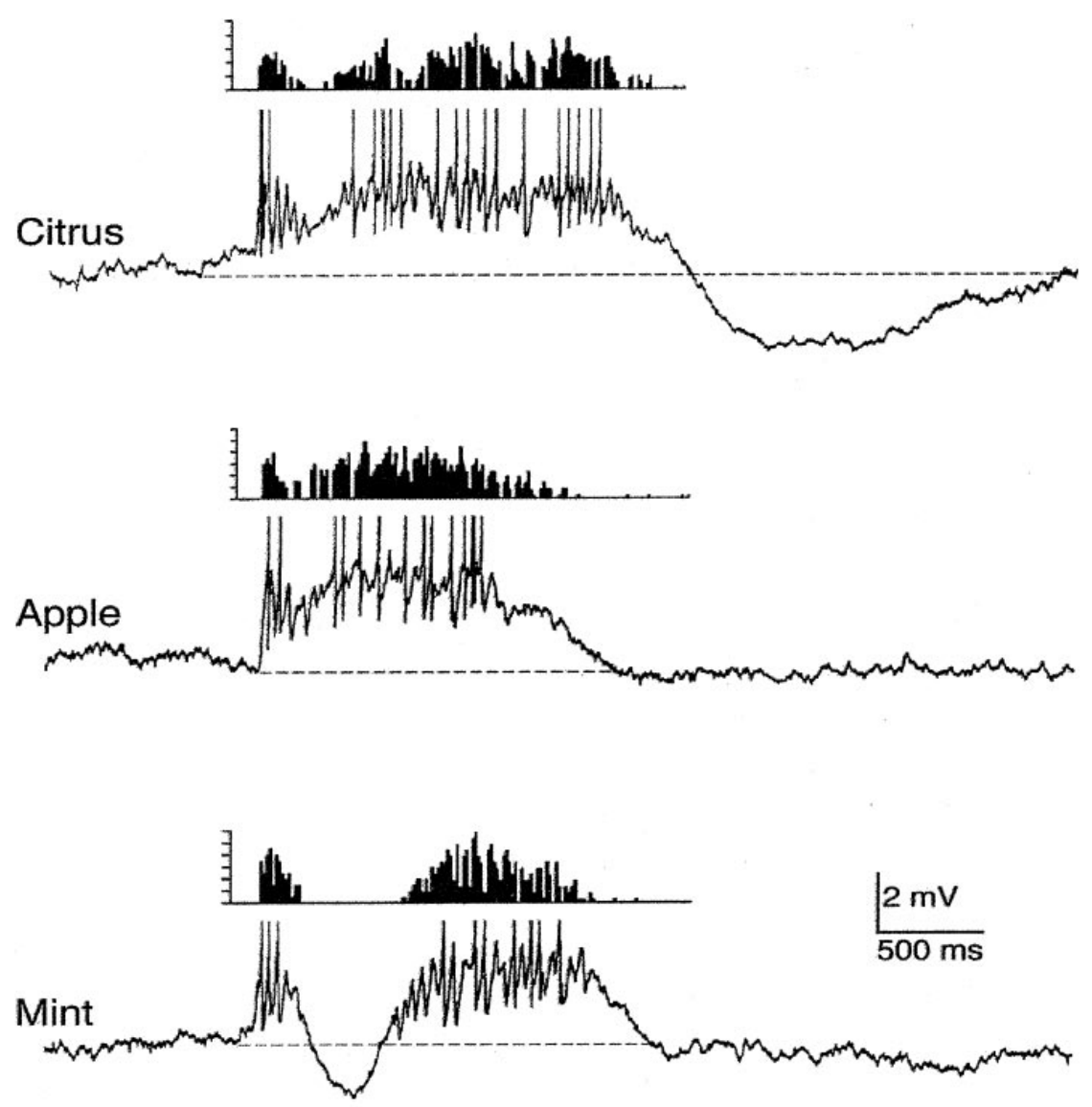

Figure 1 Intracellular recording from one locust antennal lobe projection neuron illustrating the subthreshold oscillatory activity $(50-\mathrm{ms}$ cycles) giving rise to periodic spiking superimposed on slower, odor-specific temporal-response patterns. All three recordings are from the same PN (see Laurent \& Davidowitz 1994, Laurent et al 1996).

Oscillations A typical LN or PN intracellular record shows odor-evoked subthreshold oscillations in the 20- to $30-\mathrm{Hz}$ band (Figure 1). Paired intracellular recordings show that coactive neurons within either population are phase-locked with $0^{\circ}$ mean phase, whereas LNs and PNs are phase shifted relative to each other by $90^{\circ}$ (Laurent \& Davidowitz 1994). Oscillation frequency is independent of odor identity, and air alone evokes no oscillatory activity. Because PNs project to a layered structure (the mushroom body calyx), PN coherence can be measured from local field potential (LFP) oscillations recorded there (Laurent \& Naraghi 1994). 
Whereas insect ORNs do not show odor-evoked oscillatory synchronization, sustained electrical stimulation of ORN axons causes oscillatory activity within and across PNs (Wehr \& Laurent 1999). Oscillatory synchronization of LNs and PNs is therefore the result of AL circuit dynamics, driven by ORN-evoked excitation. At rest, low-level spontaneous oscillatory activity can be detected from spectral analysis of mushroom body LFPs. Upon transsection of ORN axons, however, this spectral peak disappears. Baseline ORN activity thus sets AL dynamics on the threshold of coherent oscillatory behavior, even though basal PN activity is generally low $(1-5 \mathrm{~Hz})$.

Slow Patterning The odor-evoked responses of LNs and PNs also contain prolonged and successive periods of increased and decreased activity (Laurent 1996, Laurent \& Davidowitz 1994) shaped, in part at least, by slow, picrotoxin-insensitive synaptic inhibition (MacLeod \& Laurent 1996). These patterns are cell and odor specific (Figure 1) and are stable from trial to trial; a given odor thus evokes activity in a PN assembly whose composition changes reliably throughout the response. In addition, the action potentials produced by a PN during its odor-specific phases of activity are not necessarily all phase-locked to the LFP (Laurent et al 1996). For each odor-PN combination, however, precise and consistent epochs of phase-locked or non-phase-locked activity can usually be identified. Hence, pairwise oscillatory synchronization of PNs is generally transient (Laurent 1996, Laurent \& Davidowitz 1994), while the LFP, which reflects a larger fraction of the population's coherent activity, usually shows continuous oscillatory activity throughout the stimulus. When phase-locked, PN spikes occur within a \pm 5 -ms window around the ensemble mean. Hence, oscillatory synchronization and slow patterning together shape a complex, distributed representation in which odorspecific information appears both in the identity and in the time of recruitment and phase-locking of PNs. None of these features can be deciphered from LFPs or single-cell recordings alone.

Substrate for Odor Encoding Upon odor stimulation, large-amplitude oscillations appear in the LFP. The time scale of PN update during a response is the oscillation cycle (Wehr \& Laurent 1996). Using multiple trials with the same odor, one can assign a firing probability to each responding PN for each one of the successive cycles of the population oscillation. For a given odor, PN, and cycle, this probability can be close to 1 ; that is, each cycle of the population response contains one spike from each of several reliable PNs and less reliable spikes from many PNs with intermediate firing probabilities. The latter group is interesting because it allows one to estimate the functional coupling of PNs by measuring conditional firing probabilities. Take the example of two simultaneously recorded PNs of which one $\left(\mathrm{PN}_{1}\right)$ fired one action potential during cycle 5 in only 16 of 25 trials. Given this, what was the output of $\mathrm{PN}_{2}$ during or around cycle 5 over the nine trials when $\mathrm{PN}_{1}$ did not fire? Firing probabilities were often significantly correlated 
(positively or negatively) across PNs, so that the firing of a given PN in one trial would predict an increased or decreased firing probability in the other PN, during the same or even a different cycle of that trial (Wehr \& Laurent 1996). This indicates that each PN's firing is correlated with the present and past behavior of other PNs in the network. It does not, however, identify the causes of this correlation.

Over the few seconds that an odor response can be sustained (before ORN adaptation sets in), these patterns rarely settle or repeat themselves. Using the terminology of dynamical systems, an odor representation by PNs can be thought of as a complex trajectory through phase space (with an uncertainty given by the limited reliability of many participating PNs) with no fixed-point or limit-cycle attractor (see theory below). When the stimulus stops, PNs return to baseline firing levels either immediately or, in some instances, after a short period of deep inhibition or rapid desynchronized firing. In conclusion, specific odors at a given concentration activate odor-specific PN assemblies whose components are recruited during precise and sometimes multiple epochs of the response. Odor-specific differences can be resolved at the temporal scale of one oscillation cycle $(\sim 50$ $\mathrm{ms})$. No evidence was ever obtained for a within-cycle phase code, suggested for this or other sensory systems (Hopfield 1995, von der Malsburg \& Schneider 1986).

\section{FUNCTIONAL RELEVANCE OF OSCILLATORY SYNCHRONIZATION}

The functional relevance of these patterns was recently tested, exploiting the knowledge that oscillatory synchronization results from LN-mediated fast inhibition (MacLeod \& Laurent 1996).

\section{Selective Projection Neuron De-synchronization by Disruption of Fast Local Inhibition}

LNs make $\gamma$-aminobutyric acid-immunoreactive contacts onto both LNs and PNs (Leitch \& Laurent 1996, Malun 1991b). Among several Cl-channel or $\gamma$-aminobutyric acid receptor antagonists tested, picrotoxin was found to block selectively the oscillatory synchronization of AL neurons while sparing the slow inhibition responsible for slow patterning (MacLeod et al 1998, MacLeod \& Laurent 1996); the slow odor response patterns of individual PNs remained unchanged, except for the introduced temporal jitter of their spikes. Odor identification using temporal information contained in individual PN spike trains, for example, was not affected by desynchronization (MacLeod et al 1998). Similarly, picrotoxin never caused the appearance of PN responses to new odors (neither in locusts [MacLeod et al 1998, MacLeod \& Laurent 1996] nor in honey bees [Stopfer et al 1997]). 


\section{Behavioral Assay: Projection Neuron Synchronization and Odor Discrimination}

Evaluating the functional significance of oscillatory synchronization requires a behavioral measure of perception. Honeybees can be trained to recognize odors in a well-defined proboscis extension behavioral paradigm; after associative pairing(s) of an odor with a reward (sucrose solution or nectar), bees can predict the delivery of the reward upon presentation of the conditioned odor alone (Kuwabara 1957, Bitterman et al 1983, Smith \& Menzel 1989, Hammer \& Menzel 1995). Conditioning strength is assayed by the probability of proboscis extension during single extinction trials, carried out over a population of individuals. Because the physiological responses of bee AL neurons closely resemble those described in locusts (odor-activated oscillating neural assemblies and picrotoxin-sensitive oscillatory coherence [Stopfer et al 1997]), we could assess the putative role of PN synchronization on odor perception.

One group of bees (controls) received an application of saline into (or at the surface of) each AL; the second (picrotoxin group) received similar treatments with picrotoxin. Odor-sucrose pairing started 10 min after treatment, using an aliphatic alcohol as the conditioning stimulus $(C)$. Both groups learned to respond to $C$ equally well. Each bee's discrimination was then tested using three odorants: $C$, the alcohol used for training; $S$, a similar aliphatic alcohol; and $D$, a chemically dissimilar odorant (a terpene). These odors were chosen because bees generalize partly across the alcohols but very little from either alcohol to the terpene; this triple test thus enabled us to better assess the effects of desynchronization on odor discrimination. The control animals responded vigorously to $C$, but significantly less to $S$ and $D$, indicating that they could readily discriminate each odor. The picrotoxin group responded vigorously to $C$ but hardly at all to $D$, indicating that picrotoxin had not affected the animals' ability to memorize the association of $C$ with a reward; picrotoxin did not cause widespread generalization. This group, however, could not perform the more difficult discrimination task between $C$ and $S$. In a further study, honeybees were given two successive injections of saline or picrotoxin, one before each of the conditioning and testing periods; there were thus four groups: saline-saline, saline-picrotoxin, picrotoxin-saline, and picrotoxinpicrotoxin groups. All groups were tested with odors $C, S$, and $D$. The results were identical to those obtained previously (Stopfer et al 1997); all groups discriminated $C$ and $D$, but only the saline-saline group could distinguish $C$ from $S$ (Hosler et al 2000). We conclude that oscillatory synchronization of AL neurons is functionally relevant for tasks that require fine, but not coarse, odor discrimination (Hosler et al 2000, Stopfer et al 1997). Synchronization thus seems to enable the use of an additional coding dimension, time, which becomes important when recognition requires fine discrimination between overlapping assemblies.

Recent results obtained in mollusks support these conclusions. In Limax odors modulate the coherence of ongoing, slow $(\sim 1 \mathrm{~Hz})$ LFP oscillations in its procerebral lobe (Gelperin \& Tank 1990). These oscillations can be reversibly suppressed 
by the NO-synthase inhibitor L-NAME (Gelperin 1994). By scoring the intensity of the neural correlate of an odor-elicited behavior (tentacle positioning), odor discrimination could be assessed upon pharmacological desynchronization of procerebral-lobe networks. Saline-treated preparations were able to discriminate between similar odorants; L-NAME-treated preparations could not. The ability to recognize conditioned odorants per se, however, was not impaired by desynchronization (Teyke \& Gelperin 1999). Thus, in honeybees, AL neurons were desynchronized by blocking fast $\gamma$-aminobutyric acid-mediated inhibition; in Limax, procerebral-lobe neurons were desynchronized by blocking an NOmediated pathway. In both species, network desynchronization led to the same specific deficit-a loss of precise olfactory discrimination.

\section{Physiological Assay: Projection Neuron Synchronization and Tuning of Downstream Decoders}

The above experiments suggested that some individual neurons or groups of neurons downstream of the AL PNs are sensitive to the presence (or absence) of synchronized inputs. We identified in locusts a population of odor-sensitive neurons downstream of the AL. Rather than focus on neurons postsynaptic to PNs (e.g. the Kenyon cells of the mushroom body), we chose neurons postsynaptic to these-a subgroup of mushroom body extrinsic neurons called beta-lobe neurons ( $\beta$-LNs [MacLeod et al 1998]). The rationale was that PNs are greatly outnumbered by Kenyon cells, whereas Kenyon cells converge to many fewer $\beta$-LNs, implying the existence of a bottleneck, potentially useful as a physiological read-out of distributed PN activity.

及-Lobe Neuron Detuning by Input Desynchronization We compared $\beta$-LN odor responses recorded before and after picrotoxin injection into the AL (MacLeod et al 1998). PN de-synchronization caused, in $\beta$-LNs, the appearance of responses to odors to which they had been unresponsive before treatment. Conversely, however, desynchronization never led to the disappearance of $\beta$-LN odor responses observed before treatment. PN desynchronization also caused a loss of odor specificity in the temporal response patterns observed in controls. Hence, $\beta$-LN odor selectivity depends on PN synchronization. This shows that high-order neurons decode temporal correlations between their inputs and use them to fine-tune their sensory properties; information is contained in interneuronal temporal correlations, which cannot be deciphered from serial sampling of neurons within a population. These results also imply that some aspects at least of the information distributed across PNs eventually converge to single neurons (although it does not exclude the possibility that still more information could be retrieved from correlations across $\beta$-LNs - such tests have yet to be carried out). Finally, these data provide physiological support for the behavioral experiments in bees (Stopfer et al 1997). 
Possible Mechanisms of Coincidence Detection Because optimal odor tuning in $\beta$-LNs depends on PN synchronization, $\beta$-LNs or the neurons interposed between them and the PNs-the Kenyon cells-should possess biophysical mechanisms that favor coincident inputs (in the $20-\mathrm{Hz}$ range) over noncoincident ones. While no information exists on putative mechanisms in $\beta$-LNs, in vivo intracellular Kenyon cell recordings revealed subthreshold, voltage-dependent, tetrodotoxin-resistant properties, able to amplify PN-evoked excitatory postsynaptic potentials (EPSPs; Laurent \& Naraghi 1994). The time constant of the active EPSPs was considerably shorter than the oscillation period (due to as-yet-uncharacterized active repolarizing conductances). A Kenyon cell intracellular response to an appropriate odor thus typically contains, over its consecutive oscillation cycles, some passive (lowamplitude) EPSPs, some active (sharp, high-amplitude) EPSPs, and some action potentials, at an instantaneous frequency generally lower than the oscillation's $20 \mathrm{~Hz}$ (Laurent \& Naraghi 1994). The dendrites of Kenyon cells, by virtue of this boosting nonlinearity, could thus act as coincidence detectors of synchronized PN inputs.

\section{SHARPENING OF TEMPORAL STRUCTURE THROUGH EXPERIENCE}

\section{Response Strength and Information}

Much of sensory neurobiology depends on assuming a positive correlation between firing rate and stimulus "preference." The following study in locusts indicated that this need not be the case. Under most environmental conditions, turbulence (Murlis et al 1992) and olfactory behavior (Mellon 1997) discretize odor sampling. We found that the neural representations of odors in the AL change reliably when an animal so experiences a stimulus. By delivering odors using discrete pulses and starting from a naive state, we found that, while the response to the first pulse was the most intense, it lacked the fine temporal definition described above; neither PNs nor LNs showed periodic subthreshold activity; the LFP contained very little power in the 20- to $30-\mathrm{Hz}$ band; and oscillatory coherence between PNs or LNs was low. In summary, the synchronized, evolving ensemble response could not be identified. As odor exposures ensued, however, the ensemble response changed (Figure 2A). PN firing rates declined by half, and strong subthreshold oscillatory ripples appeared in the membrane potentials of PNs and LNs; a sharp 20- to $30-\mathrm{Hz}$ spectral peak emerged in the LFP; the odor-elicited PN spikes, although sparser, became increasingly locked to the LFP; the temporal patterns of relative PN firing (Wehr \& Laurent 1996) appeared and stabilized (Figure 2B). Within the delivery of 5 to 10 stimuli, the ensemble responses ceased to evolve further. These changes did not depend on any associative pairing of the odor with a reinforcer. They could also result from a variety of stimulation regimes 
A
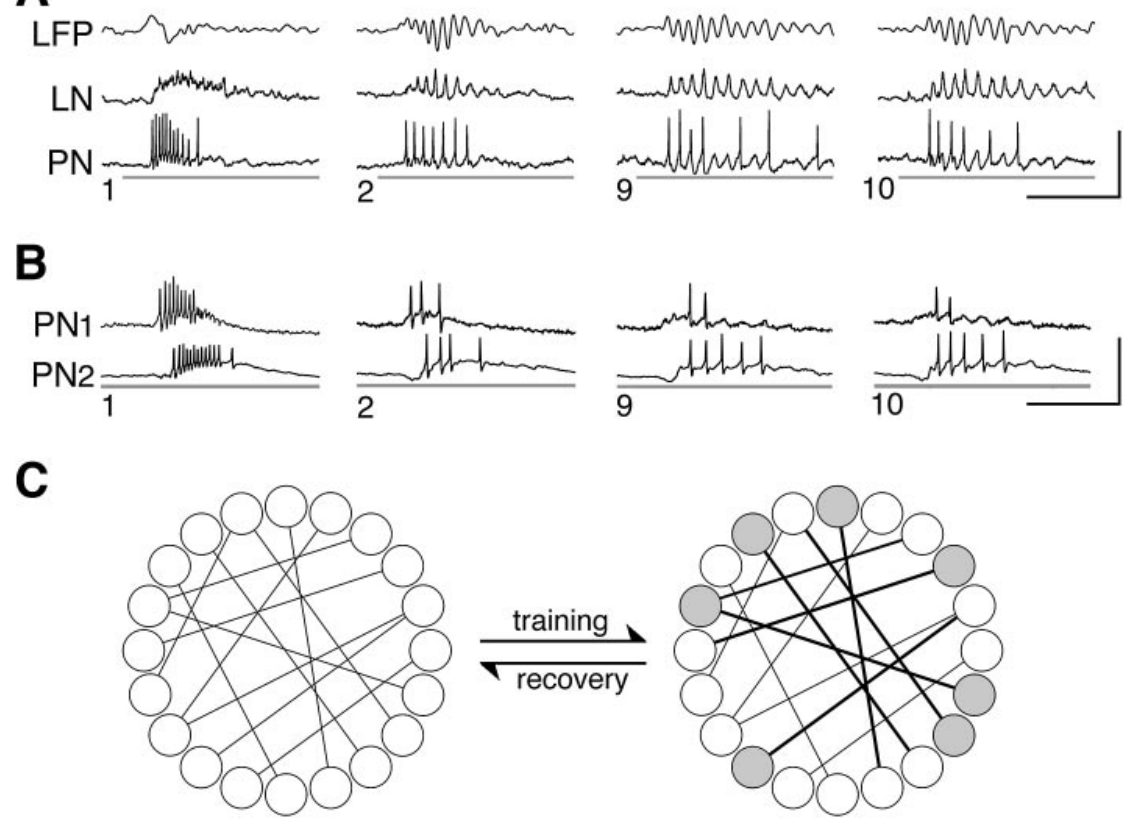

Figure 2 Nonstationarity of network dynamics. Repeated exposure to an odor causes a decrease in response intensity but an increase in oscillatory coherence and spike time precision. (A) Simultaneous local field potential (LFP) and intracellular recordings from a local $(\mathrm{LN})$ and projection $(\mathrm{PN})$ neurons during early (1-2) and later (9-10) trials. Horizontal bar indicates odor delivery. Calibration: horizontal, $300 \mathrm{~ms}$; vertical (mV), .8 (LFP), 10 (LN), and 40 (PN). (B) From a separate experiment, odor-elicited responses in two simultaneously recorded PNs illustrate increasing spike time precision over successive stimulus trials. Calibration: horizontal, $200 \mathrm{~ms}$; vertical: top trace, $70 \mathrm{mV}$; bottom trace, $40 \mathrm{mV}$. (C) Putative mechanisms for use-dependent changes in network dynamics; when the naïve AL receives repeated stimulations, only the activated neurons and/or their interconnections undergo (as yet uncharacterized) modifications (training) that endure for several minutes in the absence of further odor stimulation and spontaneously returns to the naïve state (recovery) once stimulation ends (see Stopfer \& Laurent 1999).

(e.g. interstimulus intervals of $\leq 20 \mathrm{~s}$, variable consecutive interstimulus intervals, or fewer but longer individual stimuli). Once established, the state change persisted for several minutes in the absence of further stimulation or even in the presence of interposed stimulation with other odors (Stopfer \& Laurent 1999). Thus, this evolution of olfactory network dynamics is the expression of a form of short-term memory, with a time constant of $\sim 5$ to $10 \mathrm{~min}$. A similar phenomenon was observed also in zebrafish (RW Friedrich \& G Laurent, manuscript in preparation). 


\section{Specificity}

This memory was odor specific, carrying over to chemically related, but not to chemically distinct odorants. If, for example, oscillatory synchrony had been established by presentations of 1-hexanol, then 1-octanol would immediately elicit a synchronized (although not identical) ensemble response. If, however, the new odorant were geraniol (a terpene), then a naive (strong, unsynchronized) response immediately appeared. If the new odorant were a blend containing the familiar odor, the oscillatory power fell between those of the naïve and established states. This specificity indicates that the cellular or synaptic modifications underlying the phenomenon occur only within the subset of olfactory neurons repeatedly activated by the odor used during training (Figure $2 C$ ).

\section{Sites of Changes}

In locusts, ORNs are distributed along the lengths of the antennae; we could therefore stimulate separate populations of receptors by selective odor delivery. Once strong network oscillations had been established by repeatedly stimulating one set of receptors with one odor, the same stimulus delivered to naive ORNs gave rise to a trained (sparse and coherent) response pattern (Stopfer \& Laurent 1999). Thus, the neural modifications underlying the evolution to a coherent population state must reside, at least in part, within the AL. Finally, this evolution of circuit dynamics from naïve to coherent state itself defined a consistent trajectory: two training sessions with the same odor separated by a long enough interval both produced similar "trained" PN response patterns. This self-organizing network evolution is thus deterministic and likely depends on the network's "basal" connectivity matrix (Figure $2 C$ ). The mechanisms underlying this evolution are so far unknown.

\section{Functions}

Is this sharpening of distributed odor representations relevant? Our previous work showed that the relative timing of individual PN action potentials contains information about odor identity and that oscillatory synchronization is required for refined olfactory discrimination. Because the network dynamics necessary to gain access to this relational information emerge only after some initial exposure, we predict that repeated sampling should improve behavioral odor discrimination; repeated (naturalistic) experience provides downstream decoders with more informative patterns (individual or corelational) of PN activity. Then, one might ask, why not ensure coherent and temporally precise activity at all times? We suspect that there is a trade-off between quantitative and qualitative sensitivity; unsupervised learning allows a system to have a low detectability threshold for all odors while naïve (at the expense of precision) and to have high discriminability once self-trained. When classification has been made (i.e. once the subcircuits likely to be challenged again by the next sample have been primed), all of the 
computational resources can be used to refine identification within this limited region of coding space. In the vocabulary of dynamical systems, the orbits representing individual odors progressively increase their relative distances from one another. Finally, because the effects last only a few minutes in the absence of continued stimulation, this short-term memory can be seen as a "working" feature, useful only while the animal either forms a memory or attempts to match an ongoing experience to a stored memory. What representations (early and coarse, late and refined, or possibly all) a brain ends up storing for future use remain unknown. This question probably depends primarily on associative mechanisms (Hammer \& Menzel 1995, Heisenberg et al 1985, Smith 1998) and, thus, on the precise time of pairing between odor-evoked activity and reinforcer. It would also be interesting to see whether the time constant of this memory is in any way related to the statistics of odor pulsing experienced by the animal.

\section{Multiple Time Scales of Circuit Dynamics and Consequences}

We have identified at least three time scales that are relevant for our understanding of olfactory dynamics in insects and fish (see below): a fast, periodic one (40- to $50-\mathrm{ms}$ time scale) for $20-$ to $30-\mathrm{Hz}$ oscillations; an intermediate, aperiodic one (hundreds of milliseconds) for slow response patterning; and a slow one (seconds to tens of seconds) for repeated or prolonged odor sampling. The first two are useful to characterize any individual odor response (although oscillatory power will be absent from naïve responses). The third one is useful to describe response sharpening during familiarization. This slow familiarization has several practical consequences for the experimenter. First, it suggests that multiple-trial averaging may often be inappropriate. Second, because of the nature of in vivo physiology, an experimenter usually spends much time searching for a match between neuron and stimulus; this inevitably leads to a modification of the system under study. The degree to which this search time biases the results towards a "familiarized state," not necessarily representative of a natural stimulation sequence, may thus need to be assessed. Third, it is also traditional to interleave trials while studying a neuron's response to a family of stimuli. Given that the refinement of a representation depends on frequent enough samples, some experiments may, contrary to point two above, fail to evoke the natural response evolution.

\section{DYNAMIC DECOMPOSITION OF REPRESENTATION CLUSTERS}

Our analyses of odor representations by AL PNs in insects showed that useful information is contained in individual neuron's temporal response patterns as well as in temporal correlations among them. Assume that downstream targets decode this distributed information over some limited time. Could early and late portions of a 
single response contain different information about the stimulus? Would decoders profit from reading an early or a late segment of the population response? We addressed these possibilities in zebrafish, by first comparing the global structure of amino acid odor representations by MCs, the OB's output, to those carried by their inputs, the ORNs (Friedrich \& Korsching 1997, 1998). (Given the nonstationary behavior described above and observed in fish also, we focus here on the changes that occur during single responses to familiar odors.)

\section{Dynamic Declustering of Odor Representations by Mitral-Cell Assemblies}

Amino acid representations by zebrafish ORNs are clustered: related odors evoke overlapping patterns of glomerular activity (Friedrich \& Korsching 1997), imposing that fine discrimination rely on small differences between within-cluster representations. We first examined whether clustering was found also among amino acid representations by MCs. We characterized the responses of $50 \mathrm{MCs}$ to 16 amino acids and represented each odor by a 50-dimensional vector, constructed using mean response rates. Whereas small clusters could be found, they were fewer and much less distinct than those observed among the afferent representations (RW Friedrich \& G Laurent, submitted manuscript). We then examined whether time played a role in the observed cluster reduction.

MC responses to amino acids are temporally structured (Figure $3 A$ ); while each neuron usually responds to several odors, it often responds to many with different patterns. As in the insect AL, therefore, odor-encoding neuron assemblies in the fish $\mathrm{OB}$ evolve over the stimulus duration. Clustering of odor representations by MCs was thus now analyzed over the response duration; each odor was represented by a sequence of 50-dimensional vectors, with each vector in the sequence constructed from MC firing rates calculated over a short window (e.g. $400 \mathrm{~ms}$ ), scrolled in 50- or 100-ms steps. Potential changes in odor representations by MCs could thus be examined by following the grouping of those vectors over the duration of a response (Figure $3 B$ ). This procedure revealed a dramatic evolution; initial MC responses were markedly clustered, with odor groupings identical to those observed using afferent data. Over the first $800 \mathrm{~ms}$ of the response, however, clusters disappeared; representations that were initially similar became decorrelated while dissimilar ones became slightly more correlated. Odor representations by $\mathrm{MC}$ assemblies are therefore more distinct from one another than those across ORNs, but only if the response patterns are given enough time to evolve (several hundred milliseconds); time thus seems to underlie a self-organized transformation of odor representations in the OB (RW Friedrich \& G Laurent, submitted manuscript).

\section{Functions}

Declustering did not result simply from an increase in $\mathrm{MC}$ response variability over time; the standard deviation of $\mathrm{MC}$ responses over trials decreased, if 
anything, from beginning to end of a response. In addition, odor identification by matching single-trial odor vectors to templates constructed from other single trials became more reliable as the response progressed. This improvement matched the temporal progress of declustering. Hence, representation declustering facilitates fine odor discrimination, given the uncertainties introduced by noise, by making each distributed pattern less similar to any of those evoked by other odors, including chemically related ones. In other words, redundancy is reduced by better occupying coding space. Concurrently, however, useful information about odor classes (clusters) is lost as a response progresses. The OB thus appears to solve two antagonistic problems of pattern recognition, using circuit dynamics: early responses, based (passively) on the afferents' responses, provide information relevant for perceptual grouping; later responses, resulting from afferent input and active reformatting by the $\mathrm{OB}$, provide information relevant for fine identification (RW Friedrich \& G Laurent, submitted manuscript). These results reinforce three key points introduced earlier: first, stimulus representations should be studied over cell assemblies rather than single cells; second, the resolution needed to uncover these phenomena in the $\mathrm{OB} / \mathrm{AL}$ is the single neuron; third, responses are not stationary and their odor-driven evolution is not random; rather, it appears to be optimized to facilitate recognition, without complete loss of grouping information.

Interesting studies in the frog OB (Duchamp \& Sicard 1984) seem, at first glance, to contradict our results. In these accounts, clustering of odor representations by ORNs was less than that observed across MCs. This study, however, did not use natural odor stimuli (for which each system may have been optimized) and did not analyze representations as a function of time. In addition, the clusters found each corresponded to very different chemical classes; the clusters found by us, by contrast, might be seen as subgroups within one of their clusters (amino acids). Declustering might thus be confined to representations within such larger classes, which, in fish at least, are processed within specialized subregions of the OB (Friedrich \& Korsching 1998). Indeed, in the frog, representations within a cluster appear to be less similar across MCs than across ORNs, whereas the clusters to which they belong are better defined across MCs than ORNs (Duchamp-Viret \& Duchamp 1997). It would be interesting to see these data reanalyzed over response time.

Dynamic transformations of stimulus representations occur also in other sensory systems. In monkey inferotemporal cortex, the information content of responses of face-selective neurons changes over time (Sugase et al 1999). Early response phases contain information mainly about stimulus categories (e.g. distinguishing monkey faces from human faces or abstract objects), whereas later phases contain more information about details (e.g. individual monkey faces and facial expressions). Although responses were analyzed in single neurons only, these results support the contention that circuit dynamics underlies a refinement in stimulus identification. 


\section{Mechanisms}

Noise Reduction Because this temporal declustering process was not observed among the afferents, it must result from OB circuit dynamics. Because the evolution of a representation is stimulus specific, it must rely also specifically on the stimulus. Overall, this phenomenon appears to amplify, through OB-driven processes, the small differences between odor representations first formed by afferent arrays. This suggests that stimulus noise must be efficiently controlled so as to amplify the signal preferentially. Hence, efficient noise-reducing mechanisms, possibly including the spatial averaging hypothesized earlier and chemical low-pass filters (Pelosi 1996), must exist at the earliest levels of odor processing.

Oscillatory Synchronization Zebrafish MC responses to odors also caused 20$\mathrm{Hz}$ LFP oscillations. Oscillatory synchronization, however, generally lagged the odor-induced population firing rate increase by $\sim 500 \mathrm{~ms}$. Hence, the earliest phase of odor-evoked MC activity, that for which odor clusters could be defined, was desynchronized (RW Friedrich \& G Laurent, submitted manuscript). The process of cluster dissolution, by contrast, was accompanied by the progressive development of oscillatory synchronization. Because oscillatory synchronization results from local feedback within the OB (Gray \& Skinner 1988), we conclude that the reformatting of odor representations is correlated with the development of OB circuit dynamics shaped by lateral interactions. Whether this correlation is causal remains to be determined.

Lateral Inhibition vs Redistribution One form of lateral interaction that might underlie both synchronization and declustering is GC-mediated lateral inhibition, in its classical sense (i.e. as a means to sharpen MC tuning, DeVries \& Baylor 1993, Mori \& Shepherd 1994). This hypothesis was tested in two ways. First, we assessed the sparseness (Rolls \& Tovee 1995, Vinje \& Gallant 2000) of odor representations as a function of time, over single responses. If classical lateral inhibition were responsible for declustering, one would predict $(a)$ that the average representation sparseness across MCs would be more than across ORNs (MC representations should be "sharper" than across ORNs) and (b) that the sparseness of MC representations should increase over response duration (as the better-tuned MCs inhibit those less well tuned). Neither of the two predictions was met (RW Friedrich \& G Laurent, submitted manuscript). Second, we examined the representation of all amino acids across five MCs, selected because of their similar early responses to three related amino acids; these five MCs, therefore, defined an early cluster. By simply following the "tuning" of these five cells to all 16 amino acids over the response duration, we observed no systematic sharpening or strengthening of initial responses. Rather, we noted a clear redistribution of activity of all five MCs across all 16 odors, in ways that could not be predicted from the odors' structures. The only clear trend was for a disappearance of the initial cluster, 
contradicting a prediction of the "lateral-inhibition" hypothesis. We conclude that classical lateral inhibition, the evidence for which is lacking, does not underlie declustering. To be very explicit, we do not hereby deny the existence of GCmediated inhibition; rather we mean that such inhibition does not, as far as we can assess, mediate the sharpening of single-MC odor tuning, as is usually proposed.

In short, the declustering of odor representations involves odor-induced OB activity, including lateral interactions between MCs and other neurons. The consequences of this activity are not to sharpen $\mathrm{MC}$ response profiles but rather to redistribute activity within the $\mathrm{OB}$. This redistribution is odor specific and such that late phases of the response contain the activity of more unique (i.e. more specific) MC assemblies. Response sharpness indeed increases, but only when measured over the cell population. The OB's internal circuits thus exploit stimulus-driven dynamics so as to optimize odor encoding during a response.

\section{THEORETICAL FRAMEWORK: WINNERLESS COMPETITION}

Physiological and imaging data show that odor encoding in the OB/AL is a complex, distributed dynamical process occurring over several time scales. We introduce here a theoretical framework for these observations but limit our description, for brevity's sake, to the slow evolution of OB/AL networks upon stimulation with a familiar odor (i.e. addressing deterministic, stimulus-specific slow-firing patterns in PNs and MCs, odor-specific intercell firing correlations, sustained evolution of the network during an odor, spontaneous return to baseline upon stimulus termination, and pattern reliability over multiple trials, but excluding cycle-bycycle network update and unsupervised learning over repeated presentations of a new odor).

\section{Introduction to the Language of Nonlinear Dynamics}

Nonlinear dynamics (NLD) is useful to describe neuronal-network function because it can often explain much of the observed complexity with simple models and few assumptions. It also provides a qualitative, geometric view of the behavior of a system of interdependent parts, reducing it to only a few dimensions, in which one's intuition can be trained and applied (Figure 4). The critical aspect of NLD models is that they present a theoretical foundation in which instabilities, such as those in which small changes in state are amplified rapidly, are permitted. Critically, dissipation and saturation (see below) constrain a system's motions such that a return to the starting regions of state space is possible. Consequently, a system's motion can remain within compact regions of state space and yet retain an unstable flavor. This aspect, characteristic of nonlinear systems only and well suited for olfactory processing, is central to the formal description proposed below. 
The language of dynamical systems is that of differential equations or discrete time maps. The idea of instability is easily explained with a one-dimensional differential equation in a variable $x_{i}(t)>0$,

$$
d x_{i} / d t=a S^{n} x_{i}(t)-x_{i}^{2}(t)+S_{i}^{S},
$$

where $x_{i}(t), S_{i}^{S}$, and $S^{n}$ represent, respectively, the firing rate of neuron $i$, the stimulus current provided to neuron $i$, and a trigger function for neuron $i$. Consider the simple case where $S_{i}^{s}=0$ (no external stimulus, i.e. an "autonomous" system) and $S^{n}=1$. If $a<0$, the time derivative of $x_{i}$ is negative, and $x_{i}(t)$ rapidly goes to 0 , whatever its initial value. If $a>0$, however, the state $x_{i}(t)=0$ is unstable; any state starting near $x_{i}=0$ moves away from it, grows exponentially rapidly towards $x_{i}=a$, and stops there. $x^{2}(t)$, a very simple nonlinearity, shows that instabilities in nonlinear systems can lead to change without disaster. This example illustrates a second important point: If $a>0$ and the starting state is $x(0)>a$, the system also moves to $x(t)=a$. This is the simplest example of a dynamical system with an attractor which depends on one parameter: If $a<0$, the attractor is the origin; if $a>0$, the attractor is $x=a$. In both cases, the attractors are fixed points.

The single dynamic variable $x(t)$ is not adequate to describe or understand how complex neuron assemblies might work. With more dynamical variables or degrees of freedom - that is, higher dimensional differential equations-richer behaviors may arise. For example, with three or more dynamical variables, $[x(t), y(t), z(t), \ldots]$, in addition to time-independent states $(x=0$ or $x=a$ in the above example) and periodic oscillations $\{[x(t+T), y(t+T), z(t+T), \ldots]=$ $[x(t), y(t), z(t), \ldots]\}$, nonperiodic behaviors become possible; these characterize chaotic motions of a system. In each of these cases, instabilities in some region of state space (e.g. $x=0$ when $a>0$ ) play a central role. With chaotic oscillations, for example, there is an infinite set of unstable points, and the system evolves from one unstable region of state space to another. We can classify these instabilities by an exponent, indicative of how rapidly two nearby states move away from each other. In our simple example, near $x=0$ and when $a>0$, the solution to the state evolution equation is $x(t) \approx x(0) e^{a t}$. The growth index $a$ is called a Lyapunov or instability exponent. In a multidimensional system, there is an index for each dimension of state space. In chaotic, dissipative, and long-term motions, one or more of these exponents is positive, some are negative, and the sum of all is always negative.

\section{Olfactory Networks as Dissipative and Active Systems}

Plasticity notwithstanding, we observe that, to be useful to the animal, odor representation should be reproducible, that is, by and large insensitive to the network's initial state (internal noise). In the language of NLD, this is possible only if the system is strongly dissipative, in other words, if it can rapidly forget its initial state. In dissipative systems, the initial phase volume is rapidly compressed and all trajectories converge to attractors (fixed points, closed trajectories or limit cycles, strange attractors, or other specific trajectories such as homoclinic/heteroclinic 
trajectories). On the other hand, a useful olfactory system should be sensitive to small variations in the inputs, so that fine discriminations between similar but not identical stimuli are possible. How can both conditions coexist? This is only possible if a system is active; an active system uses external sources of energy to increase a small distance between initial states (representations) caused by similar stimuli, independent of the initial state of the network. Because the system is active, small initial differences between representations can grow rapidly over time. Time thus plays a critical role in separating representations. The OB/AL should therefore behave as active, nonlinear, dissipative systems. Although many existing models of early olfactory systems satisfy these conditions, these models present limitations, briefly examined below.

\section{Coding with Attractors}

The most common nonlinear dynamical models of early olfactory processing (representation and recognition) lead to the idea of "coding with attractors" ("Hopfield nets" [Cohen \& Grossberg 1982, Hopfield 1982]). In these models, each stimulus or odor is represented by a specific behavior (attractor) of the neural network. The behavior expressed depends on the connections among the network's elements. The number of different attractors determines the number of different stimuli that the system can represent or recognize. Because many stable states must coexist, the system is called multistable (Figure $4 a$ ). Each attractor possesses its own basin of attraction, and different basins are separated from each other by boundaries whose shapes can be complex (usually fractal). The main idea behind a Hopfield network is that, during a learning stage, a long-acting stimulus is used to modify specific sets of connections until a steady-state behavior (attractor) specific for that stimulus is obtained. Through training, each stimulus thus generates its own attractor. After learning, this trained, autonomous system becomes multistable (the unstimulated system possesses several basins of attraction); for recognition, future incoming stimuli will simply play the role of initial conditions. Hence, although a Hopfield network is a dynamical system, it is static (or stationary) after convergence. Time does not play an intrinsic role in the encoding or decoding of the input. This appears to conflict with experimental results on early olfactory processing, as reported above.

A very important feature of such a network is that, during recognition, the evolution of the system within a basin of attraction is resistant to corruption of the input (noise or missing features). Conversely, however, the number $m$ of stimuli (attractors) allowed in a system of $N$ neurons is relatively limited: $m<0.14 N$ (Hertz et al 1991). This limit is due to increasingly complex boundaries between basins of attraction as the number of desired stimuli rises; small mistakes, such as ones resulting from noise in the initial conditions, lead to increasing error rates. Such systems therefore have hard capacity limits. For these reasons, we think that such models, although very important from a conceptual point of view, do not capture some essential features of biological olfactory systems. 


\section{Population Models and Chaos}

Walter Freeman and collaborators have, over the years, followed a different approach, merging experiments and NLD theory. They developed dynamical models that simulate the global behavior of neuron population (Freeman 1975, 1992, 2000) as manifested in EEG recordings from the mammalian OB. These models of population dynamics (called "mesoscopic" models because of their intermediate scale) reproduce several important aspects of EEG data. For example, at rest, the models produce irregular oscillations whose origin lies in dynamical chaos. The mathematical signature of this behavior is called a strange attractor. There is now much experimental evidence that EEG time series can indeed sometimes be interpreted as expressions of dynamical chaos (e.g. Elbert et al 1994). In addition, Freeman's models generate transient and reproducible waveforms that uniquely depend on the stimulus; they can thus be used for recognition. These models therefore have a true temporal component. On the other hand, they do not explicitly model individual neurons, groups of synchronized neurons, or network topology (Freeman 1987, Yao \& Freeman 1990). They propose to explain macroscopic signals (as indicated by EEG) whose functional relevance per se is debatable and, in any case, difficult to assess without explicit description of their underlying causes. Hence, we find it difficult to transpose these spatiotemporal models into the biological realm.

\section{A New Theoretical Framework: "Winnerless Competition"}

The simple model in Equation 1 embodies part of what is needed for a dynamical system with input-specific behavior. If $x_{i}(t)$ and $a$, respectively, represent neuron $i$ 's firing rate and the state of the input ( $a \leq 0$, stimulus off; $a \geq 0$, stimulus on), then the neuron can move between 0 (stimulus off) and $a$ (stimulus on). This system, however, has a very limited capacity. To encode many stimuli, the dynamics should allow stimulus-specific trajectories $x_{i}(t)$ in state space. We introduce the conditions required for such dynamics.

First, many neurons are required. For the sake of simplicity, $x_{i}(t)$ will represent the firing rate of the $i$ th neuron or, collectively, that of the $i$ th group of synchronized neurons. The functional unit of the network is thus either the neuron or a small group of coactive, synchronized neurons. The identity of the elements of a group may change over time, in which case the identity of the group will similarly evolve. The mechanisms underlying this evolution will become clear as we proceed.

Second, these neurons (or groups) must interact at least in part through inhibitory connections. If $x_{i}(t)$ and $x_{j}(t)$ characterize the activities of groups $i$ and $j$, respectively, the system's behavior can be described by

$$
\frac{d x_{i}}{d t}=x_{i}(t)\left[1-\sum_{j=1}^{N} \rho_{i j} x_{j}(t)\right]+S_{i}^{s},
$$

where $\rho_{i j}>0$ characterizes the strength of inhibition of $i$ by $j$ and $S_{i}^{s}$ is the input current contributed by stimulus $s$ to $i$. The simplest dynamics arise if these 
inhibitory connections are symmetrical $\left(\rho_{i j}=\rho_{j i}\right)$. In this case, the autonomous system $\left(S_{i}^{s}=0\right)$ is "potential" or gradient, and Equation 2 can be rewritten as

$$
\frac{d x_{i}}{d t}=-\frac{\partial F}{\partial x_{i}}
$$

and

$$
\frac{d F}{d t} \leq 0,
$$

where $F$ is the free energy or Lyapunov function. Equation 4 implies that the system cannot generate complex temporal patterns; rather, free energy must decrease monotonically along any trajectory in state space or remain constant; the system can only move to a local minimum and stay there. If $\rho_{i j}>\rho_{i i}$, the free-energy landscape for a system with $N$ neurons (or groups) has $N$ or fewer minima and behaves as a multistable Hopfield network. If $\rho_{i j}<\rho_{i i}$, the system has only one global attractor, corresponding to simultaneous activity of all $\mathrm{N}$ elements ("weak competition") (Figure 4b).

If connections are not symmetrical, two cases arise, depending on the nature of the asymmetries. If the asymmetry is only partial (e.g. for $N=3, \rho_{12} \sim \rho_{21}>1$; $\left.\rho_{13}, \rho_{23}>1 ; \rho_{31}, \rho_{32}<1\right)$, only one attractor, corresponding to the activity of one neuron, will result (Figure $4 c$ ). This is a "winner-take-all" circuit (e.g. Yuille \& Grzywacs 1989). These and Hopfield-like networks are well studied in dynamical systems theory (Morse-Smale systems [e.g. see Guckenheimer \& Holmes 1983]) and generally display behaviors too simple to account for experimental data. If the asymmetries are cyclic $\left(\rho_{i j}>\rho_{i i}\right.$, but $\rho_{j i}<\rho_{i i}$; e.g. for $N=3, \rho_{12}, \rho_{23}$, and $\rho_{31}>1$, and $\rho_{21}, \rho_{32}$, and $\rho_{13}<1$ ) however, more interesting behaviors arise in which activity "bounces off" between neurons (groups), in a stimulus-dependent manner (Figure 4d). We call this behavior "winnerless competition". Its geometrical description is a heteroclinic orbit, that is, a trajectory linking quasi-stationary states within phase space (Figure 5). One critical point, developed further below, is that heteroclinic orbits are very sensitive to the stimulus. Consequently, each orbit can be associated with a stimulus and thus "encode" it. A second (related) critical point is that the state space of the system is enlarged upon reception of the input. We propose that this form of nonlinear dynamical behavior best describes odor-evoked activity in the early olfactory system. How and why does it work?

\section{Closed Topology}

An important condition for this form of dynamical behavior is that the network topology (connectivity) be closed. This condition is fulfilled by AL and OB circuits, whose synaptology is well known (Masson \& Mustaparta 1990, Scott 1986, Shipley \& Ennis 1996); a mitral cell, for example, will excite other mitral or granule cells which in turn will feed back onto it through mono- or polysynaptic pathways. In addition, if reciprocal connections exist between local inhibitory neurons and the principal neurons that excite them, those inhibitory connections 
should not be so powerful as to cause, on their own, an immediate silencing of the principal neurons. This condition appears to be fulfilled in the locust AL, where no evidence for functional reciprocal inhibitory connections has been found either morphologically (Leitch \& Laurent 1996) or physiologically from recordings of connected pairs (MacLeod \& Laurent 1996; G Laurent, personal observation). In the vertebrate $\mathrm{OB}$, well-documented reciprocal inhibition via granule cells never prevents prolonged MC firing caused either by an odor stimulus or by direct depolarizing current injection (Isaacson 1999, Isaacson \& Strowbridge 1998, Nicoll $\&$ Jahr 1982). These features are important because they act to disperse activity across the neural assembly once a few elements have been activated by a stimulus.

Activity thus proceeds across parts of the network by sequentially activating and deactivating subgroups of neurons. Individual neurons can belong to several subgroups recruited at different times during the stimulation, and different subgroups join and depart over successive, sometimes overlapping epochs. Hence, at any time during the stimulation, each neuron may receive inputs from the afferents or from other neurons in the network and thereby contribute, by its own activity combined with that of the afferents, to the excitation and inhibition of other neurons. This activation path within the network (or, correspondingly, this heteroclinic orbit in the state space that describes it) is determined by and thus "represents" the stimulus. Once the stimulus ceases (either because it was withdrawn or because of receptor adaptation), each active neuron returns to its baseline activity, controlled by intrinsic properties, basal-connection strengths, and noise; correspondingly, the system returns to the neighborhood of the origin in state space. A stimulus can thus be thought of as a perturbational or informational signal that reorganizes the global attractor in a stimulus-specific manner, forcing the system to evolve through state space along a complex, but deterministic path joining unstable "saddle states." Once the stimulus disappears, the system resets itself autonomously, because no stable attractor other than the resting state could be found. Hence, the system's only attractors are the stimulus-evoked heteroclinic orbits (one for each stimulus) or, when no stimulus is present, the origin.

\section{Advantages of Winnerless Competition}

This dynamical-systems description of early olfactory processing took shape as a means to explain experimental data (Laurent et al 1996, Wehr \& Laurent 1996; RW Friedrich \& G Laurent, submitted manuscript). Although its global features appear to match the data, our description should also provide insights as to what is gained by such dynamical behavior.

Global Stability Winnerless competition provides global stability to the representation. Although each neuron or subgroup participating in the representation might, left to its own devices, wander during a response, the effect of the population behavior is to provide local and temporary stability to each, by attracting them to the neighborhood of a succession of quasi-stable saddle states. Each neuron's local activity thus inherits the global stability of the heteroclinic orbit defined by 
the system. This stability can be verified by examining the reproducibility of stimulus-evoked trajectories in model networks of simplified LNs and PNs (MI Rabinovich, R Huerta, A Volkovskii, HDI Abarbanel, G Laurent, in preparation) or in experimental data (Laurent et al 1996, Wehr \& Laurent 1996).

Sensitivity Such dynamics are very sensitive to the forcing stimulus. This is because the heteroclinic linking of a specific set of saddle points is always unique. Two like stimuli, activating greatly overlapping subsets of a network, may thus become easily separated because small initial differences will become amplified in time. This feature is central to our experimental observations (RW Friedrich \& G Laurent, submitted manuscript; Stopfer \& Laurent 1999) and is characteristic of an active, dissipative, nonlinear dynamical system. We do not know yet, however, whether divergence can be optimized by fine-tuning of the internal connectivity. Intermittent-stimulation experiments indeed suggest that short-term changes can increase the separability of odor representations (Stopfer \& Laurent 1999). The rules for such unsupervised improvements are so far unknown.

Capacity A heteroclinic (spatiotemporal) representation provides greatly increased capacity to the system. Because sequences of activity are combinatorial across neurons and time, overlap between representations can be reduced, and the distance in phase space between orbits can be increased, thus reducing the effects of noise. This feature, given by a dynamic representation, is absent from a Hopfield-like network, where the intricacy of the boundaries between neighboring basins of attraction can easily lead to classification errors.

Implementation Details Finally, does the expression of such dynamics depend on the details of the network or neuron implementation? Indeed, to be general, this theory should accommodate the many idiosyncrasies of OBs, ALs, and other equivalent circuits and rely on broad organizational principles (Hildebrand \& Shepherd 1997). Preliminary tests indicate that this is indeed the case; winnerless competition arises from a few simple and biologically relevant rules.

\section{GENERAL CONCLUSIONS}

The thrust of this review is that the transfer of odor-evoked signals from receptors to $\mathrm{OB} / \mathrm{AL}$ circuits is accompanied by a fundamental reformatting of odor representations. This reshaping of odor codes results from the internal connectivity of early olfactory circuits and from the global dynamics that these connections produce. This reshaping is useful in that it removes ambiguities about stimulus identity, exploiting time as an additional coding dimension. We do not mean to suggest, however, that time is the only coding dimension for olfaction. Rather we tried to explain that neural identity and temporal recruitment of neurons are, in 
these circuits at least, two sides of the same coin; because the OB/ALs are networks of interacting neurons and because odor stimuli are usually sustained, the structure of odor-encoding neural assemblies is shaped both by the stimulus and by the distributed patterns forced by the stimulus. We propose that odor-encoding neural assemblies in the OB/AL are dynamical, by virtue of the physical laws that govern such systems' behavior (winnerless competition).

This perspective has several practical correlates. First, it suggests that, because information is distributed across neural assemblies, traditional measures such as single-neuron tuning should be appropriately weighted. By analogy, take a symphonic piece in which the piccolo plays one measure only, but one whose role is critical to the melodic line that emerges from the orchestra. The relevance of the piccolo to the global output is accurately conveyed not by the strength of its contribution but by its relation to the other instruments' output. Melodic representation is, in this context, a property of the ensemble. Second, this perspective (and our results) casts doubts on the traditional ("single-neuron") functional interpretation of lateral inhibitory contacts. Third, our results demonstrate the importance of system nonstationarity at several time scales. The critical observation is that, over time and without supervision, olfactory networks become increasingly attuned to a stimulus's precise identity.

Finally, we must emphasize that many important aspects (e.g. intensity coding, decoding mechanisms, and the role of high-level features such as expectation) remain to be studied in detail, incorporated, and tested within this framework. Similarly, physiological studies on larger vertebrates such as reptiles and mammals show a greater phenomenological complexity. These features will eventually also need to be accounted for. Our hope is that a computational/theoretical perspective combined with experiments on small and tractable systems can help both in framing questions of general relevance and in designing more direct experimental tests.

\section{ACKNOWLEDGMENTS}

The work described here was supported by the NIDCD, the Sloan Center for Theoretical Neuroscience at Caltech, the Keck Foundation, the McKnight Foundation (GL), and the US Department of Energy, Office of Basic Energy Sciences, Division of Engineering and Geosciences (HDIA, MIR).

\section{Visit the Annual Reviews home page at www.AnnualReviews.org}

\section{LITERATURE CITED}

Adrian ED. 1942. Olfactory reactions in the brain of the hedgehog. J. Physiol. 100:459-73

Adrian ED. 1950. The electrical activity of the olfactory bulb. Electroencephalogr. Clin. Neurophysiol. 2:377-88
Adrian ED. 1953. Sensory messages and sensation. The responses of the olfactory organ to different smells. Acta Physiol. Scand. 29:514

Aroniadou-Anderjaska V, Ennis M, Shipley M. 
1999. Dendrodendritic recurrent excitation in mitral cells of the rat olfactory bulb. J. Neurophysiol. 82:489-94

Attneave F. 1954. Informational aspects of visual perception. Psychol. Rev. 61:183-93

Axel R. 1995. The molecular logic of smell. Sci. Am. 273:130-37

Barlow H. 1969. Pattern recognition and the responses of sensory neurones. Ann. NY Acad. Sci. 156:872-81

Beuerman R. 1975. Slow potentials of the turtle olfactory bulb in response to odor stimulation of the nose. Brain Res. 97:61-78

Bitterman M, Menzel R, Fietz A, Schaefer S. 1983. Classical conditioning of proboscis extension in honeybees (Apis mellifera). $J$. Comp. Psychol. 97:107-19

Boeckh J, Tolbert LP. 1993. Synaptic organization and development of the antennal lobe in insects. Microsc. Res. Tech. 24:260-80

Bozza TC, Kauer JS. 1997. Odorant response properties of convergent olfactory receptor neurons. J. Neurosci. 18:4560-69

Buck LB. 1995. Unraveling chemosensory diversity. Cell 83:349-52

Buck LB. 1996. Information coding in the vertebrate olfactory system. Annu. Rev. Neurosci. 19:517-44

Buck LB, Axel R. 1991. A novel multigene family may encode odorant receptors: a molecular basis for odor recognition. Cell 65:175-87

Burrows M, Boeckh J, Esslen J. 1982. Physiological and morphological properties of interneurons in the deutocerebrum of male cockroaches with responses to female pheromones. J. Comp. Physiol. A 145:447-57

Cain WS, Potts BC. 1996. Switch and bait: probing the discriminative basis of odor identification via recognition memory. Chem. Senses 21:35-44

Chen WR, Midtgaard J, Shepherd GM. 1997. Forward and backward propagation of dendritic impulses and their synaptic control in mitral cells. Science 278:463-67

Cinelli AR, Hamilton KA, Kauer JS. 1995. Salamander olfactory bulb neuronal activity observed by video rate, voltage-sensitive dye imaging. III. Spatial and temporal properties of responses evoked by odorant stimulation. J. Neurophysiol. 73:2053-71

Cinelli AR, Kauer JS. 1992. Voltage-sensitive dyes and functional activity in the olfactory pathway. Annu. Rev. Neurosci. 15:321-51

Cohen M, Grossberg S. 1982. Neural networks and physical systems with emergent computational abilities. Proc. Natl. Acad. Sci. USA 79:2554-58

DeVries SH, Baylor DA. 1993. Synaptic circuitry of the retina and olfactory bulb. Cell 10:139-49

Dorries KM, Kauer JS. 2000. Relationships between odor-elicited oscillations in the salamander olfactory epithelium and olfactory bulb. J. Neurophysiol. 83:754-65

Dryer L, Graziadei PPC. 1994. Mitral cell dendrites: a comparative approach. Anat. Embryol. 189:91-106

Duchamp A. 1982. Electrophysiological responses of olfactory bulb neurons to odour stimuli in the frog: a comparison with receptor cells. Chem. Senses 7:191-210

Duchamp A, Revial M, Holley A, MacLeod P. 1974. Odor discrimination by frog olfactory receptors. Chem. Senses 1:213-33

Duchamp A, Sicard G. 1984. Odour discrimination by olfactory bulb neurons: statistical analysis of electrophysiological responses and comparison with odour discrimination by receptor cells. Chem. Senses 9:1-14

Duchamp-Viret P, Duchamp A. 1997. Odor processing in the frog olfactory system. Prog. Neurobiol. 53:561-602

Dulac C. 1997. Molecular biology of pheromone perception in mammals. Semin. Cell Dev. Biol. 8:197-205

Elbert T, Ray W, Kowalik A, Skinner J, Graf K, Birbaumer N. 1994. Chaos and physiology: deterministic chaos in excitable cell assemblies. Physiol. Rev. 74:1-47

Field D. 1987. Reactions between the statistics of natural images and the response properties of cortical cells. J. Opt. Soc. Am. A 4:237994 
Field D. 1994. What is the goal of sensory coding? Neural Comput. 6:559-601

Freeman WJ. 1975. Mass Action in the Nervous System. New York: Academic

Freeman WJ. 1978. Spatial properties of an EEG event in the olfactory bulb and cortex. Electroencephalogr. Clin. Neurophysiol. 44:586-605

Freeman WJ. 1987. Simulation of chaotic EEG patterns with a dynamic model of the olfactory system. Biol. Cybern. 56:139-50

Freeman WJ. 1992. Tutorial on neurobiology: from single neurons to brain chaos. Int. J. Bifurcation Chaos 2:451-82

Freeman WJ. 2000. Neurodynamics: An Exploration in Mesoscopic Brain Dynamics. London: Springer. 397 pp.

Freeman WJ, Skarda CA. 1985. Spatial EEG patterns, non-linear dynamics and perception: the neo-Sherringtonian view. Brain Res. 357:147-75

Friedrich RW, Korsching SI. 1997. Combinatorial and chemotopic odorant coding in the zebrafish olfactory bulb visualized by optical imaging. Neuron 18:737-52

Friedrich RW, Korsching SI. 1998. Chemotopic, combinatorial and noncombinatorial odorant representations in the olfactory bulb revealed using a voltage-sensitive axon tracer. J. Neurosci. 18:9977-88

Gelperin A. 1994. Nitric oxide mediates network oscillations of olfactory interneurons in a terrestrial mollusc. Nature 369:6163

Gelperin A, Tank DW. 1990. Odour-modulated collective network oscillations of olfactory interneurons in a terrestrial mollusc. Nature 345:437-40

Getchell TV. 1986. Functional properties of vertebrate olfactory receptor neurons. Physiol. Rev. 66:772-818

Gray C. 1994. Synchronous oscillations in neuronal systems: mechanisms and function. $J$. Comput. Neurosci. 1:11-38

Gray C, Skinner J. 1988. Centrifugal regulation of neuronal activity in the olfactory bulb of the waking rabbit as revealed by reversible cryogenic blockade. Exp. Brain Res. 69:37886

Guckenheimer J, Holmes P. 1983. Nonlinear Oscillations, Dynamical Systems and Bifurcations of Vector Fields, pp. 64-453. New York: Springer-Verlag

Haberly LB. 1990. Comparative aspects of olfactory cortex. In Cerebral Cortex, ed. EG Jones, A Peters, pp. 137-66. New York: Plenum

Hamilton KA, Kauer JS. 1989. Patterns of intracellular potentials in salamander mitral/tufted cells in response to odor stimulation. J. Neurophysiol. 62:609-25

Hammer M. 1993. An identified neuron mediates the unconditioned stimulus in associative olfactory learning in honeybees. Nature 366:59-63

Hammer M, Menzel R. 1995. Learning and memory in the honeybee. J. Neurosci. 15:1617-30

Heinbockel T, Kloppenburg P, Hildebrand J. 1998. Pheromone-evoked potentials and oscillations in the antennal lobes of the sphinx moth Manduca sexta. J. Comp. Physiol. A 182:702-14

Heisenberg M. 1998. What do the mushroom bodies do for the insect brain? An introduction. Learn. Mem. 5:1-10

Heisenberg M, Borst A, Wagner S, Byers D. 1985. Drosophila mushroom body mutants are deficient in olfactory learning. J. Neurogenet. 2:1-30

Hertz J, Krogh A, Palmer R. 1991. Introduction to the Theory of Neural Computation. Santa Fe: Addison-Wesley

Hildebrand JG, Shepherd GM. 1997. Mechanisms of olfactory discrimination: converging evidence for common principles across phyla. Annu. Rev. Neurosci. 20:595-631

Hopfield JJ. 1982. Neural networks and physical systems with emergent computational abilities. Proc. Natl. Acad. Sci. USA 79:2554-58

Hopfield JJ. 1995. Pattern recognition computation using action potential timing for stimulus representation. Nature 376:33-36 
Hosler J, Buxton K, Smith B. 2000. Impairment of olfactory discrimination by blockade of GABA and nitric oxide activity in the honey bee antennal lobes. Behav. Neurosci. In press

Hubel D, Wiesel T. 1968. Receptive fields and functional architecture of monkey striate cortex. J. Physiol. 195:215-43

Hughes J, Mazurowski J. 1962. Studies on the supracallosial mesial cortex of unanesthetized, conscious mammals. II. Monkeys. B. Responses from the olfactory bulb. Electroencephalogr. Clin. Neurophysiol. 14:63545

Imamura K, Mataga N, Mori K. 1992. Coding of odor molecules by mitral/tufted cells in rabbit olfactory bulb. I. Aliphatic compounds. J. Neurophysiol. 68:1986-2002

Isaacson JS. 1999. Glutamate spillover mediates excitatory transmission in the rat olfactory bulb. Neuron 23:377-84

Isaacson JS, Strowbridge BW. 1998. Olfactory reciprocal synapses: dendritic signaling in the CNS. Neuron 20:749-61

Joerges J, Küttner A, Galizia CG, Menzel R. 1997. Representations of odours and odour mixtures visualized in the honeybee brain. Nature 387:285-88

Kang J, Caprio J. 1995. In vivo responses of single olfactory receptor neurons in the channel catfish, Ictalurus punctatus. J. Neurophysiol. 73:172-77

Katoh K, Koshimoto H, Tani A, Mori K. 1993. Coding of odor molecules by mitral/tufted cells in rabbit olfactory bulb. II. Aromatic compounds. J. Neurophysiol. 70:2161-75

Kauer JS. 1987. Coding in the olfactory system. In Neurobiology of Taste and Smell, ed. TE Finger, WL Silver, pp. 205-22. New York: Wiley

Kauer JS. 1991. Contributions of topography and parallel processing to odor coding in the vertebrate olfactory pathway. Trends $\mathrm{Neu}$ rosci. 14:79-85

Kauer JS, Moulton D. 1974. Responses of olfactory bulb neurones to odour stimulation of small nasal areas in the salamander. J. Physiol. 243:717-37
Kay L, Freeman W. 1998. Bidirectional processing in the olfactory-limbic axis during olfactory behavior. Behav. Neurosci. 112: 541-53

Kay L, Laurent G. 1999. Odor- and contextdependent modulation of mitral cell activity in behaving rats. Nat. Neurosci. 2:1003-9

Keverne E. 1999. The vomeronasal organ. Science 286:716-20

Kuwabara M. 1957. Bildung des bedingten Reflexes von Pavlovs Typus bei der Honigbiene, Apis mellifica. J. Fac. Sci. Hokkaido Univ. Ser. VI Zool. 13:458-64

Lam Y-W, Cohen L, Wachowiak M, Zochowski M. 1999. Odors elicit three different oscillations in the turtle olfactory bulb. J. Neurosci. 19:749-62

Laurent G. 1996. Dynamical representation of odors by oscillating and evolving neural assemblies. Trends Neurosci. 19:489-96

Laurent G. 1999. A systems perspective on early olfactory coding. Science 286:723-28

Laurent G, Davidowitz H. 1994. Encoding of olfactory information with oscillating neural assemblies. Science 265:1872-75

Laurent G, Naraghi M. 1994. Odorant-induced oscillations in the mushroom bodies of the locust. J. Neurosci. 14:2993-3004

Laurent G, Wehr M, Davidowitz H. 1996. Temporal representations of odors in an olfactory network. J. Neurosci. 16:3837-47

Lawless H. 1997. Olfactory Psychophysics. San Diego, CA: Academic

Leitch B, Laurent G. 1996. GABAergic synapses in the antennal lobe and mushroom body of the locust olfactory system. J. Comp. Neurol. 372:487-514

Leveteau J, MacLeod P. 1966. Olfactory discrimination in the rabbit olfactory glomerulus. Science 153:175-76

Lin D, Wang F, Lowe G, Gold G, Axel R, et al. 2000. Formation of precise connections in the olfactory bulb occurs in the absence of odorant-evoked neuronal activity. Neuron 26:69-80

MacLeod K, Bäcker A, Laurent G. 1998. Who reads temporal information contained across 
synchronized and oscillatory spike trains? Nature 395:693-98

MacLeod K, Laurent G. 1996. Distinct mechanisms for synchronization and temporal patterning of odor-encoding neural assemblies. Science 274:976-79

Macrides F, Chorover SL. 1972. Olfactory bulb units: activity correlated with inhalation cycles and odor quality. Science 185:84-87

Malnic B, Hirono J, Sato T, Buck LB. 1999. Combinatorial receptor codes for odors. Cell 96:713-23

Malun D. 1991a. Inventory and distribution of synapses of identified uniglomerular projection neurons in the antennal lobe of Periplaneta americana. J. Comp. Neurol. 305:34860

Malun D. 1991b. Synaptic relationships between GABA-immunoreactive neurons and an identified uniglomerular projection neuron in the antennal lobe of Periplaneta americana: a double labeling electron microscopic study. Histochemistry 96:197-207

Masson C, Mustaparta H. 1990. Chemical information processing in the olfactory system of insects. Physiol. Rev. 70:199-245

Mellon D. 1997. Physiological characterization of antennular flicking reflexes in the crayfish.

J. Comp. Physiol. A. 180:553-65

Menzel R. 1987. Memory traces in honeybees. In Neurobiology and Behaviour of Honeybees, ed. R Menzel, A Mercer, pp. 310-25. Berlin: Springer-Verlag

Meredith M. 1986. Patterned response to odor in mammalian olfactory bulb: the influence of intensity. J. Neurophysiol. 56:572-97

Mombaerts P, Wang F, Dulac C, Chao SK, Nemes A, et al. 1996a. Visualizing an olfactory sensory map. Cell 87:675-86

Mombaerts P, Wang F, Dulac C, Vassar R, Chao SK, et al. 1996b. The molecular biology of olfactory perception. Cold Spring Harb. Symp. Quant. Biol. 61:135-45

Mori K, Kishi K, Ojima H. 1983. Distribution of dendrites of mitral, displaced mitral, tufted, and granule cells in the rabbit olfactory bulb. J. Comp. Neurol. 219:339-55
Mori K, Mataga N, Imamura K. 1992. Differential specificities of single mitral cells in rabbit olfactory bulb for a homologous series of fatty acid odor molecules. J. Neurophysiol. 67:786-89

Mori K, Nowycky MC, Shepherd GM. 1981. Analysis of synaptic potentials in mitral cells in the isolated turtle olfactory bulb. J. Physiol. 314:295-309

Mori K, Shepherd GM. 1994. Emerging principles of molecular signal processing by mitral/tufted cells in the olfactory bulb. Semin. Cell Biol. 5:65-74

Moulton DG. 1967. Spatio-temporal patterning of response in the olfactory system. In Olfaction and Taste, vol. II, ed. T Hayashi, pp. 109-16. Oxford/New York: Pergamon

Murlis J, Elkington J, Carde R. 1992. Odor plumes and how insects use them. Annu. Rev. Entomol. 37:505-32

Ngai J, Chess A, Dowling MM, Necles N, Macagno ER, Axel R. 1993. Coding of olfactory information: topography of odorant receptor expression in the catfish olfactory epithelium. Cell 72:667-80

Nicoll R. 1971a. Pharmacological evidence for GABA as the transmitter in granule cell inhibition in the olfactory bulb. Brain Res. 35:137-49

Nicoll R. 1971b. Recurrent excitation of secondary olfactory neurons: a possible mechanism for signal amplification. Science 171:824-25

Nicoll RA, Jahr CE. 1982. Self-excitation of olfactory bulb neurones. Nature 296:44144

Nieuwenhuys R. 1967. Comparative anatomy of olfactory centers and tracts. Prog. Brain Res. 23:1-63

Olshausen BA, Field DJ. 1996. Emergence of simple-cell receptive field properties by learning a sparse code for natural images. Nature 381:607-9

Ottoson D. 1959. Comparison of slow potentials evoked in the frog's nasal mucosa and olfactory bulb by natural stimulation. Acta Physiol. Scand. 47:149-59 
Pager J. 1983. Unit responses changing with behavioral outcome in the olfactory bulb of unrestrained rats. Brain Res. 289:8798

Pelosi P. 1996. Perireceptor events in olfaction. J. Neurobiol. 30:3-19

Rall W, Shepherd G. 1968. Theoretical reconstruction of field potentials and dendrodendritic synaptic interactions in olfactory bulb. J. Neurophysiol. 31:884-915

Ressler KJ, Sullivan SL, Buck LB. 1993. A zonal organization of odorant receptor gene expression in the olfactory epithelium. Cell 73:597-609

Rolls ET, Tovee MJ. 1995. Sparseness of the neuronal representation of stimuli in the primate temporal visual-cortex. J. Neurophysiol. 73:713-26

Rubin BD, Katz LC. 1999. Optical imaging of odorant representations in the mammalian olfactory bulb. Neuron 23:499-511

Rudderman D. 1994. Statistics of natural images. Network 5:517-48

Satou M. 1990. Synaptic organization, local neuronal circuitry, and functional segregation of the teleost olfactory bulb. Prog. Neurobiol. 34:115-42

Scott J. 1986. The olfactory bulb and central pathways. Experientia 42:223-32

Scott JW, Wellis DP, Riggott MJ, Bounviso N. 1993. Functional organization of the main olfactory bulb. Microsc. Res. Tech. 24:14256

Shadlen MN, Newsome WT. 1995. Is there signal in the noise? Curr. Opin. Neurobiol. 5:248-50

Shepherd GM. 1993. Principles of specificity and redundancy underlying the organization of the olfactory system. Microsc. Res. Tech. 24:106-12

Shepherd GM. 1994. Discrimination of molecular signals by the olfactory receptor neuron. Neuron 13:771-90

Shipley MT, Ennis M. 1996. Functional organization of olfactory system. J. Neurobiol. 30:123-76

Singer W, Gray C. 1995. Visual feature integra- tion and the temporal correlation hypothesis. Annu. Rev. Neurosci. 18:555-86

Smith BH. 1998. Analysis of interactions in binary odorant mixtures. Physiol. Behav. 65:397-407

Smith BH, Menzel R. 1989. The use of electromyogram recordings to quantify odorant discrimination in the honeybee, Apis mellifera. J. Insect Physiol. 35:369-75

Stewart WB, Kauer JS, Shepherd GM. 1979. Functional organization of rat olfactory bulb analysed by the 2-deoxyglucose method. $J$. Comp. Neurol. 185:715-34

Stopfer M, Bhagavan S, Smith BH, Laurent G. 1997. Impaired odour discrimination on desynchronization of odour-encoding neural assemblies. Nature 390:70-74

Stopfer M, Laurent G. 1999. Short-term memory in olfactory network dynamics. Nature 402:664-68

Strausfeld N, Hansen L, Li Y, Gomez R, Ito K. 1998. Evolution, discovery and interpretations of arthropod mushroom bodies. Learn. Mem. 5:11-37

Sugase Y, Yamane S, Ueno S, Kawano K. 1999. Global and fine information coded by single neurons in the temporal visual cortex. Nature 400:869-73

Sun X, Tolbert L, Hildebrand J. 1993. Ramification pattern and ultrastructural characteristics of the serotonin-immunoreactive neuron in the antennal lobe of the moth Manduca sexta. J. Comp. Neurol. 338:5-16

Teyke T, Gelperin A. 1999. Olfactory oscillations augment odor discrimination, not odor identification by Limax CNS. NeuroReport 10:1-8

Vassar R, Chao SK, Sitcheran R, Nunez JM, Vosshall LB, Axel R. 1994. Topographic organization of sensory projections to the olfactory bulb. Cell 79:981-91

Vinje WE, Gallant JL. 2000. Sparse coding and decorrelation in primary visual cortex during natural vision. Science 287:1273-76

von der Malsburg C, Schneider W. 1986. A neural cocktail-party processor. Biol. Cybern. 54:29-40 
Wang F, Nemes A, Mendelsohn M, Axel R. 1998. Odorant receptors govern the formation of a precise topographic map. Cell 93:47-60

Wehr M, Laurent G. 1996 Odor encoding by temporal sequences of firing in oscillating neural assemblies. Nature 384:162-66

Wehr M, Laurent G. 1999. Relationship between afferent and central temporal patterns in the locust olfacory system. J. Neurosci. 19:381-90

Wellis DP, Scott JW Harrison TA. 1989 Discrimination among odorants by single neurons of the rat olfactory bulb. J. Neurophysiol. 61:1161-77

Weth F, Nadler W, Korsching S. 1996. Nested expression domains for odorant receptors in zebrafish olfactory epithelium. Proc. Natl. Acad. Sci. USA 93:13321-26

Yao Y, Freeman WJ. 1990. Model of biological pattern recognition with spatially chaotic dynamics. Neural Netw. 3:153-70

Yokoi M, Mori K, Nakanishi S. 1995. Refinement of odor molecule tuning by dendrodendritic synaptic inhibition in the olfactory bulb. Proc. Natl. Acad. Sci. USA 92:3371-75

Yuilli A, Grzywacs N. 1989. A winner-take-all mechanism based on presynaptic inhibition. Neural Comput. 1:334-47

Zheng C, Feinstein P, Bozza T, Rodriguez I, Mombaerts P. 2000. Peripheral olfactory projections are differentially affected in mice deficient in a cyclic nucleotide-gated channel subunit. Neuron 26:81-91 

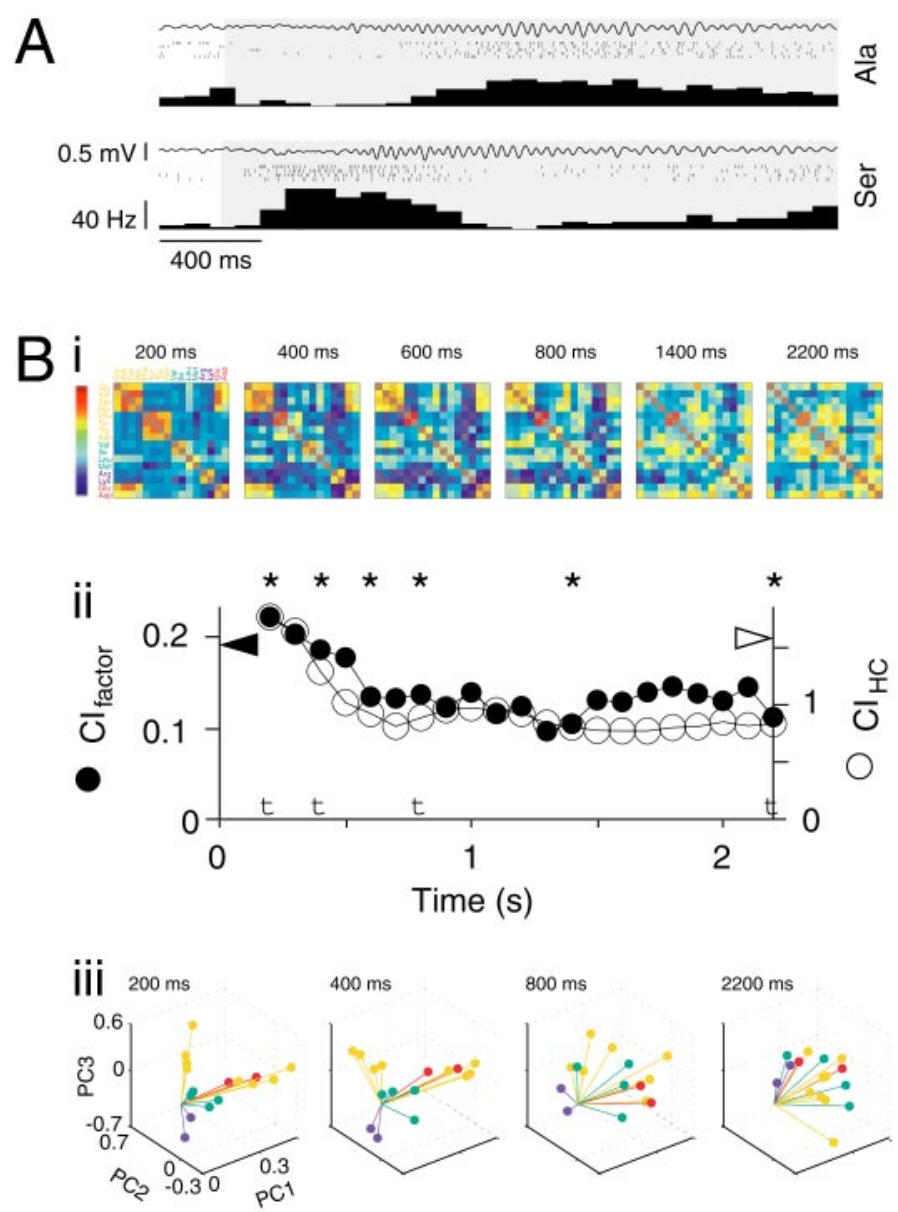

Figure 3 Temporal declustering of odor representations by MC assemblies in the zebrafish olfactory bulb. (A) Responses of one MC to 2 of 16 amino acids tested. Top, LFP; middle, spike raster; bottom, peristimulus time histogram. Gray shadow, odor stimulation. Note the odor-specific temporal-firing patterns and the late onset of LFP oscillations. (B) Quantification of declustering over time. $i$, correlation matrix plotting similarity between odor representations, as measured from a 50-mitral-cell assembly (see RW Friedrich \& G Laurent, submitted manuscript). Each matrix is constructed from firing rates measured over a 400-ms-long epoch, starting at odor onset. Times above matrices indicate middle of each epoch (corresponding to ${ }^{*}$ in $i i$ ). Note clear odor clusters along diagonal in leftmost matrix and their progressive dissolution. $i i$, clustering indices (see above reference for details) as functions of time throughout odor response; declustering reaches steady-state after $\sim 800 \mathrm{~ms}$. iii, principal component analysis of same data. Projection of odor representations in the space defined by first three principal components for four epochs ( $t$ in part $i i)$. Note dissolution of vector groups. 


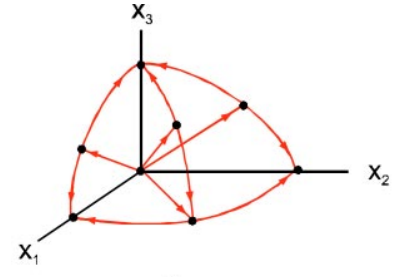

a

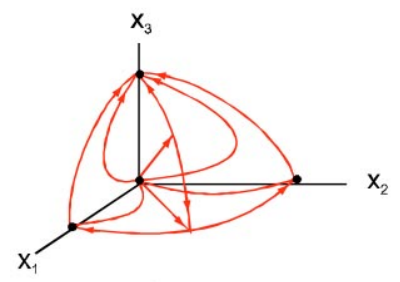

$\mathrm{C}$

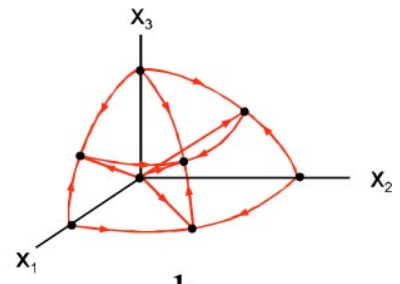

$\mathrm{b}$

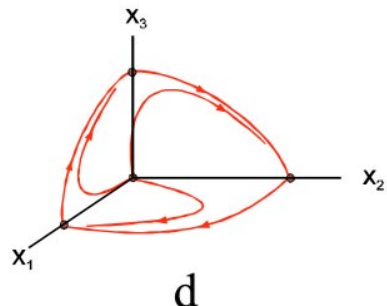

d

Figure 4 Competitive systems dynamics. Schematic illustrations of four types of behavior. $(a)$ Multistability, $(b)$ weak competition, $(c)$ winner take all, $(d)$ winnerless competition.

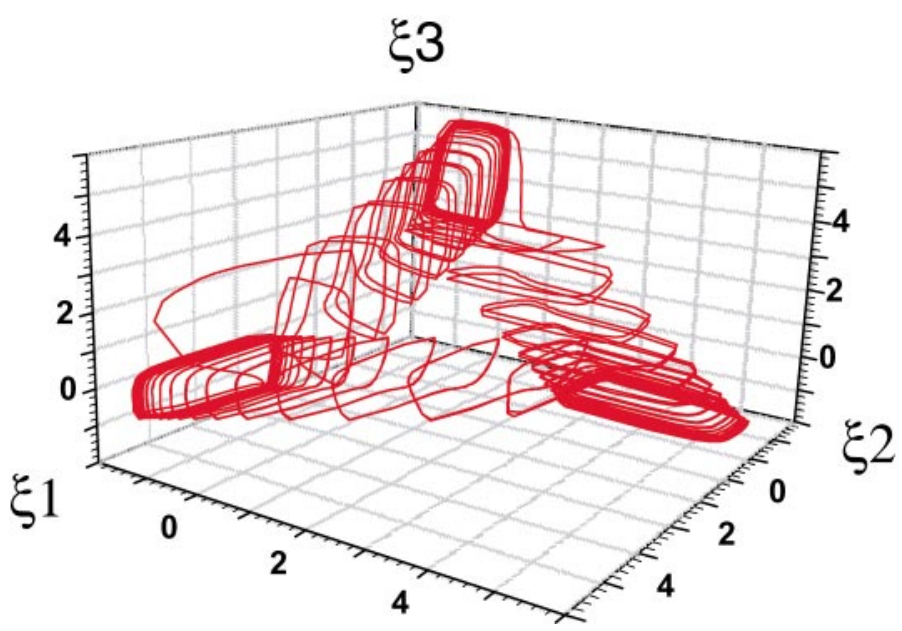

Figure 5 Heteroclinic loop for a simple network of three oscillating projection neurons (PNs), activated by a given input pattern. Each axis maps the activity of one PN through a state variable $\xi$. The sequence connecting saddle limit cycles and the intervals between them are functions of the stimulus. A different stimulus would thus be represented by a different orbit and thus, a different temporal pattern of activation of the PNs. 


\section{CONTENTS}

PDZ DOMAINS AND THE ORGANIZATION OF

SUPRAMOLECULAR COMPLEXES, Morgan Sheng, Carlo Sala

THE ROLE AND REGULATION OF ADENOSINE IN THE CENTRAL

TOUCH AND GO: Decision-Making Mechanisms in Somatosensation,

SYNAPTIC MODIFICATION BY CORRELATED ACTIVITY: Hebb"s

Postulate Revisited, Guo-qiang Bi, Mu-ming Poo

AN INTEGRATIVE THEORY OF PREFRONTAL CORTEX

FUNCTION, Earl K. Miller, Jonathan D. Cohen

THE PHYSIOLOGY OF STEREOPSIS, B. G. Cumming, G. C.

DeAngelis

PARANEOPLASTIC NEUROLOGIC DISEASE ANTIGENS: RNA-

Binding Proteins and Signaling Proteins in Neuronal Degeneration, Kiran

Musunuru, Robert B. Darnell

ODOR ENCODING AS AN ACTIVE, DYNAMICAL PROCESS:

Experiments, Computation, and Theory, Gilles Laurent, Mark Stopfer,

Rainer W Friedrich, Misha I Rabinovich, Alexander Volkovskii, Henry

DI Abarbanel

PROTEIN SYNTHESIS AT SYNAPTIC SITES ON DENDRITES,

Oswald Steward, Erin M. Schuman

SIGNALING AND TRANSCRIPTIONAL MECHANISMS IN

PITUITARY DEVELOPMENT, Jeremy S. Dasen, Michael G. Rosenfeld NEUROPEPTIDES AND THE INTEGRATION OF MOTOR

RESPONSES TO DEHYDRATION , Alan G. Watts

THE DEVELOPMENTAL BIOLOGY OF BRAIN TUMORS, Robert

Wechsler-Reya, Matthew P. Scott

TO EAT OR TO SLEEP? OREXIN IN THE REGULATION OF

FEEDING AND WAKEFULNESS, Jon T. Willie, Richard M. Chemelli, Christopher M. Sinton, Masashi Yanagisawa

SPATIAL PROCESSING IN THE BRAIN: The Activity of Hippocampal

Place Cells, Phillip J. Best, Aaron M. White, Ali Minai

385

429

THE VANILLOID RECEPTOR: A Molecular Gateway to the Pain

Pathway, Michael J Caterina, David Julius

PRION DISEASES OF HUMANS AND ANIMALS: Their Causes and

Molecular Basis, John Collinge

VIKTOR HAMBURGER AND RITA LEVI-MONTALCINI: The Path to

the Discovery of Nerve Growth Factor, W. Maxwell Cowan

459

EARLY DAYS OF THE NERVE GROWTH FACTOR PROTEINS,

Eric M. Shooter

487

SEQUENTIAL ORGANIZATION OF MULTIPLE MOVEMENTS:

Involvement of Cortical Motor Areas, Jun Tanji

519

551

601

631

INFLUENCE OF DENDRITIC CONDUCTANCES ON THE INPUT-

OUTPUT PROPERTIES OF NEURONS, Alex Reyes 
NEUROTROPHINS: Roles in Neuronal Development and Function, Eric

$J$ Huang, Louis F Reichardt

677

CONTRIBUTIONS OF THE MEDULLARY RAPHE AND

VENTROMEDIAL RETICULAR REGION TO PAIN MODULATION

737

AND OTHER HOMEOSTATIC FUNCTIONS, Peggy Mason

ACTIVATION, DEACTIVATION, AND ADAPTATION IN

VERTEBRATE PHOTORECEPTOR CELLS, Marie E Burns, Denis A

Baylor

779

ACTIVITY-DEPENDENT SPINAL CORD PLASTICITY IN HEALTH

AND DISEASE, Jonathan $R$ Wolpaw, Ann $M$ Tennissen

807

QUANTITATIVE GENETICS AND MOUSE BEHAVIOR, Jeanne $M$

Wehner, Richard A Radcliffe, Barbara J Bowers

845

EARLY ANTERIOR/POSTERIOR PATTERNING OF THE

MIDBRAIN AND CEREBELLUM, Aimin Liu, Alexandra L Joyner

869

NEUROBIOLOGY OF PAVLOVIAN FEAR CONDITIONING, Stephen

Maren

897

$\{\{$ alpha $\}$-LATROTOXIN AND ITS RECEPTORS: Neurexins and

CIRL/Latrophilins, Thomas C Südhof

IMAGING AND CODING IN THE OLFACTORY SYSTEM, John $S$

Kauer, Joel White

THE ROLE OF THE CEREBELLUM IN VOLUNTARY EYE

MOVEMENTS, Farrel R Robinson, Albert F Fuchs

ROLE OF THE REELIN SIGNALING PATHWAY IN CENTRAL

NERVOUS SYSTEM DEVELOPMENT, Dennis S Rice, Tom Curran

HUMAN BRAIN MALFORMATIONS AND THEIR LESSONS FOR

NEURONAL MIGRATION, M Elizabeth Ross, Christopher A Walsh

MORPHOLOGICAL CHANGES IN DENDRITIC SPINES

ASSOCIATED WITH LONG-TERM SYNAPTIC PLASTICITY, Rafael

Yuste, Tobias Bonhoeffer

STOPPING TIME: The Genetics of Fly and Mouse Circadian Clocks,

1091

Ravi Allada, Patrick Emery, Joseph S. Takahashi, Michael Rosbash

NEURODEGENERATIVE TAUOPATHIES, Virginia M-Y Lee, Michel

Goedert, John Q Trojanowski

MATERNAL CARE, GENE EXPRESSION, AND THE

TRANSMISSION OF INDIVIDUAL DIFFERENCES IN STRESS

933

963

981

1005

1041

REACTIVITY ACROSS GENERATIONS, Michael J Meaney

NATURAL IMAGE STATISTICS AND NEURAL

REPRESENTATION, Eero P Simoncelli, Bruno A Olshausen

1071

Nerve Growth Factor Signaling, Neuroprotection, and Neural Repair,

Michael V Sofroniew, Charles L Howe, William C Mobley

112

FLIES, GENES, AND LEARNING, Scott Waddell, William G Quinn

1161

1193

1217

1283 